ARTICLE

\title{
Structural and mechanistic basis of capsule O-acetylation in Neisseria meningitidis serogroup A
}

Timm Fiebig (10 1,7凶, Johannes T. Cramer (1) 2,7 ${ }^{2}$ Andrea Bethe ${ }^{1}$, Petra Baruch ${ }^{3}$, Ute Curth ${ }^{3}$, Jana I. Führing 1,4, Falk F. R. Buettner (10 ${ }^{1}$, Ulrich Vogel ${ }^{5}$, Mario Schubert (iD ${ }^{6}$, Roman Fedorov ${ }^{3}$ \& Martina Mühlenhoff (iD) ${ }^{1 凶}$

O-Acetylation of the capsular polysaccharide (CPS) of Neisseria meningitidis serogroup A $(\mathrm{NmA})$ is critical for the induction of functional immune responses, making this modification mandatory for CPS-based anti-NmA vaccines. Using comprehensive NMR studies, we demonstrate that $\mathrm{O}$-acetylation stabilizes the labile anomeric phosphodiester-linkages of the NmA-CPS and occurs in position C3 and C4 of the $\mathrm{N}$-acetylmannosamine units due to enzymatic transfer and non-enzymatic ester migration, respectively. To shed light on the enzymatic transfer mechanism, we solved the crystal structure of the capsule O-acetyltransferase $\mathrm{CsaC}$ in its apo and acceptor-bound form and of the CsaC-H228A mutant as trapped acetyl-enzyme adduct in complex with CoA. Together with the results of a comprehensive mutagenesis study, the reported structures explain the strict regioselectivity of $\mathrm{CsaC}$ and provide insight into the catalytic mechanism, which relies on an unexpected Glnextension of a classical Ser-His-Asp triad, embedded in an $\alpha / \beta$-hydrolase fold.

\footnotetext{
${ }^{1}$ Institute of Clinical Biochemistry, Hannover Medical School, Hannover, Germany. ${ }^{2}$ Institute for Virology, Hannover Medical School, Hannover, Germany. ${ }^{3}$ Institute for Biophysical Chemistry, Hannover Medical School, Hannover, Germany. ${ }^{4}$ Fraunhofer International Consortium for Anti-Infective Research (iCAIR), Hannover, Germany. ${ }^{5}$ Institute for Hygiene and Microbiology, University of Würzburg, Würzburg, Germany. ${ }^{6}$ Department of Biosciences, University of Salzburg, Salzburg, Austria. ${ }^{7}$ These authors contributed equally: Timm Fiebig, Johannes T. Cramer. ${ }^{凶}$ email: Fiebig.Timm@mh-hannover.de; Muehlenhoff.Martina@mh-hannover.de
} 
N eisseria meningitidis ( $\mathrm{Nm}$ ) is an encapsulated, strictly human pathogen, which remains an important cause of bacterial meningitis and septicemia, especially in the African meningitis belt and in India ${ }^{1}$. The capsular polysaccharide (CPS) is the major virulence factor and serves as the basis for potent anti-meningococcal glycoconjugate vaccines ${ }^{2}$. Licensed formulations are available against serogroups A, C, W and $\mathrm{Y}$, including an affordable monovalent anti-NmA vaccine developed for combatting the devastating meningococcal disease outbreaks in sub-Saharan Africa ${ }^{3-5}$. The NmA-CPS is joint by phosphodiester bridges ${ }^{6,7}$ that are highly susceptible to hydrolysis at elevated temperatures, which has major implications for the shelf-life and storage conditions of anti-NmA vaccines in tropical and subtropical areas ${ }^{4,8}$.

Composed of $[\rightarrow 6)-\alpha-D-M a n N A c-\left(1 \rightarrow \mathrm{OPO}_{3}-\rightarrow\right]$ repeating units, the NmA-CPS backbone is heavily modified by variable degrees of $\mathrm{O}$-acetylation at position $\mathrm{C} 3$ and $\mathrm{C} 4^{5,9-12}$. Although four of the six clinically relevant $\mathrm{Nm}$ serogroups express $O$-acetylated capsules ${ }^{7,12}$, it is only the NmA-CPS, for which $O$-acetylation is mandatory to induce an effective immune response after vaccination ${ }^{12,13}$. Consequently, all vaccine formulations against $\mathrm{NmA}$ contain $\mathrm{O}$-acetylated $\mathrm{CPS}$ and precise knowledge on the biosynthesis, intramolecular distribution, and functional impact of this modification is crucial for our understanding of meningococcal biology and tailored vaccine development. The key enzyme is the $\mathrm{O}$-acetyltransferase CsaC (MynC) encoded in region A of the CPS gene cluster ${ }^{14,15}$. Gene deletion completely abolished capsule $O$-acetylation and biochemical studies identified the assembled polymer as acceptor substrate $^{14,16}$, suggesting that $O$-acetylation occurs co- or postsynthetically (Fig. 1).

Aiming at a cost-effective synthesis platform that omits the cultivation of biohazardous material, we have recently established reaction conditions for the cell-free in vitro production of $\mathrm{NmA}$ CPS. Usage of the recombinant biosynthetic enzymes CsaA, CsaB and CsaC (Fig. 1) allowed efficient in vitro assembly of the polymer and subsequent $O$-acetylation of $88 \%$ of the repeating units $^{16,17}$. This approach, however, yielded exclusively 3-Oacetylated polymer and to date, the mechanism of CsaC-mediated $\mathrm{O}$-acetylation and the origin of 4-O-acetylation are unknown. Moreover, structural information on meningococcal capsule $O$-acetyltransferases is, so far, only available for CssF (OatWY) of $\mathrm{NmW}$ and NmY. This enzyme belongs to the left-handed $\beta$-helix family of acyltransferases ${ }^{18}$ and shares no sequence similarity with CsaC.

Herein, we report three crystal structures of $\mathrm{CsaC}$, in an apo and two substrate complexed forms, and provide mechanistic insight into $\mathrm{NmA}$ capsule $\mathrm{O}$-acetylation. Supported by a comprehensive two-dimensional (2D) NMR characterization of the CPS, we demonstrate that the $\mathrm{CsaC}$-mediated reaction is regioselective for $\mathrm{O} 3$ and that modification of $\mathrm{O} 4$ results from spontaneous $O$-acetyl migration. A comparative analysis of modified and unmodified NmA-CPS demonstrates that $O$-acetylation considerably stabilizes the polymer, allowing hydrolysis only to take place between non-O-acetylated sugar residues.

\section{Results}

4-O-Acetylation of $\mathrm{NmA}$ CPS is due to acetyl migration. For natural NmA-CPS contained in vaccines, total $O$-acetylation levels between $68-92 \%$ have been reported, of which roughly $10 \%$ is $4-O$-acetylation ${ }^{5,11,12}$. To investigate, whether similar levels can be achieved by in vitro $O$-acetylation, we incubated $\mathrm{CsaC}$ with 1 $\mathrm{mg}$ of non-O-acetylated polymer in the presence of different donor (acetyl-CoA) to acceptor ratios, with each ManNAc moiety a

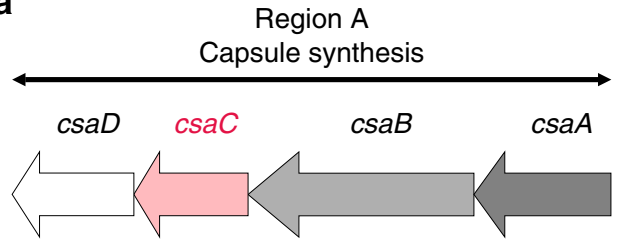

b
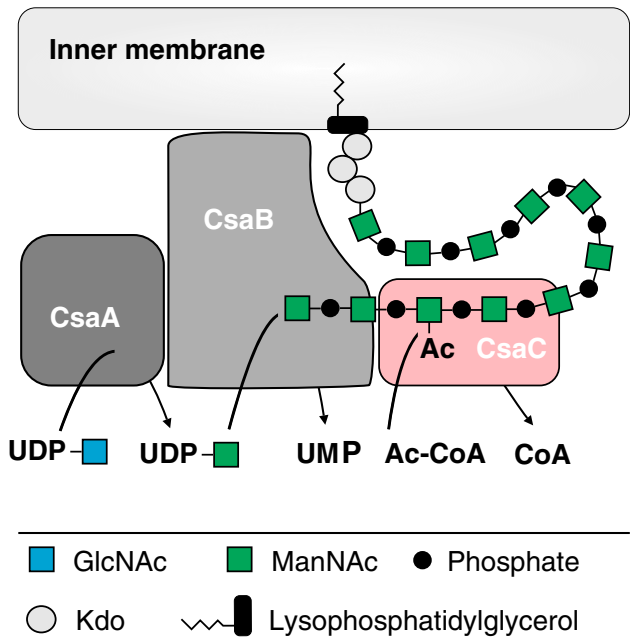

Fig. 1 Capsule biosynthesis in Neisseria meningitidis serogroup A. a Schematic representation of region $\mathrm{A}$ of the capsule gene cluster of Neisseria meningitidis serogroup $A^{15}$ encoding the UDP-N-acetyl-D-glucosamine-2epimerase $\mathrm{CsaA}$, the capsule polymerase $\mathrm{CsaB}$, the capsule $\mathrm{O}$-acetyltransferase $\mathrm{CsaC}$, and a protein of unknown function ( $\mathrm{CsaD}$ ) predicted to be involved in CPS transport ${ }^{15}$. $\mathbf{b}$ Scheme summarizing the key steps of capsule biosynthesis in serogroup A. The UDP-N-acetyl-D-glucosamine-2-epimerase CsaA catalyzes the epimerization of UDP-GIcNAc to UDP-ManNAc. The capsule polymerase CsaB transfers ManNAc-1-phosphate units from UDP-ManNAc onto the nonreducing end of the growing polysaccharide chain $[\rightarrow 6)-\alpha-D-M a n N A c-(1 \rightarrow$ $\left.\mathrm{OPO}_{3}^{-} \rightarrow\right]$. The $\mathrm{O}$-acetyltransferase $\mathrm{CsaC}$ transfers acetyl-groups from acetylCoA onto the ManNAc units of assembled polysaccharide ${ }^{14,16}$. In all group 2 capsule biosynthesis complexes, the biosynthesis enzymes are believed to be membrane associated and the polymer is built upon a highly conserved acceptor consisting of lysophosphatidylglycerol and 3-deoxy-D-manno-oct-2ulosonic acid $(K d o)^{67}$.

being considered as one acceptor site. Changing the ratio from 1:1 to $4: 1$ indeed increased the overall $O$-acetylation level from 70 to 92\% (Supplementary Fig. 1a, b). Since acetyl groups can migrate between vicinal diols in a $\mathrm{pH}$-dependent manner ${ }^{7}$, we next aimed at analyzing in vitro 3-O-acetylated $\mathrm{NmA}$-CPS under different $\mathrm{pH}$ conditions to induce a potential 3,4-ester migration. Production of a larger batch of material for this experiment $(20 \mathrm{mg})$ yielded 3-O-acetylated polymer, which already contained $4 \%$ of 4 $O$-acetylation (Fig. 2b, blue spectrum), indicating that acetyl migration occurred to a small extent already during the extended preparation time. Consequently, we aimed at reproducing these conditions in our experiment: The polymer was dissolved in phosphate buffer ( $\mathrm{pH}$ 7.0) to mimic the enzymatic synthesis conditions, or in water $(\mathrm{pH} 5.6)$ to simulate the dialysis step of our processing protocol. The percentage of 3-O- and 4-O-acetylated moieties was determined by ${ }^{1} \mathrm{H}$ NMR based on their characteristic $\mathrm{H} 2$ chemical shifts ${ }^{7}$. Already after one day, 4-Oacetylation was increased from 4 to $14 \%$ in phosphate buffer and remained constant upon prolonged incubation (Fig. 2b, red spectrum). In contrast, $O$-acetyl migration in water progressed 
a

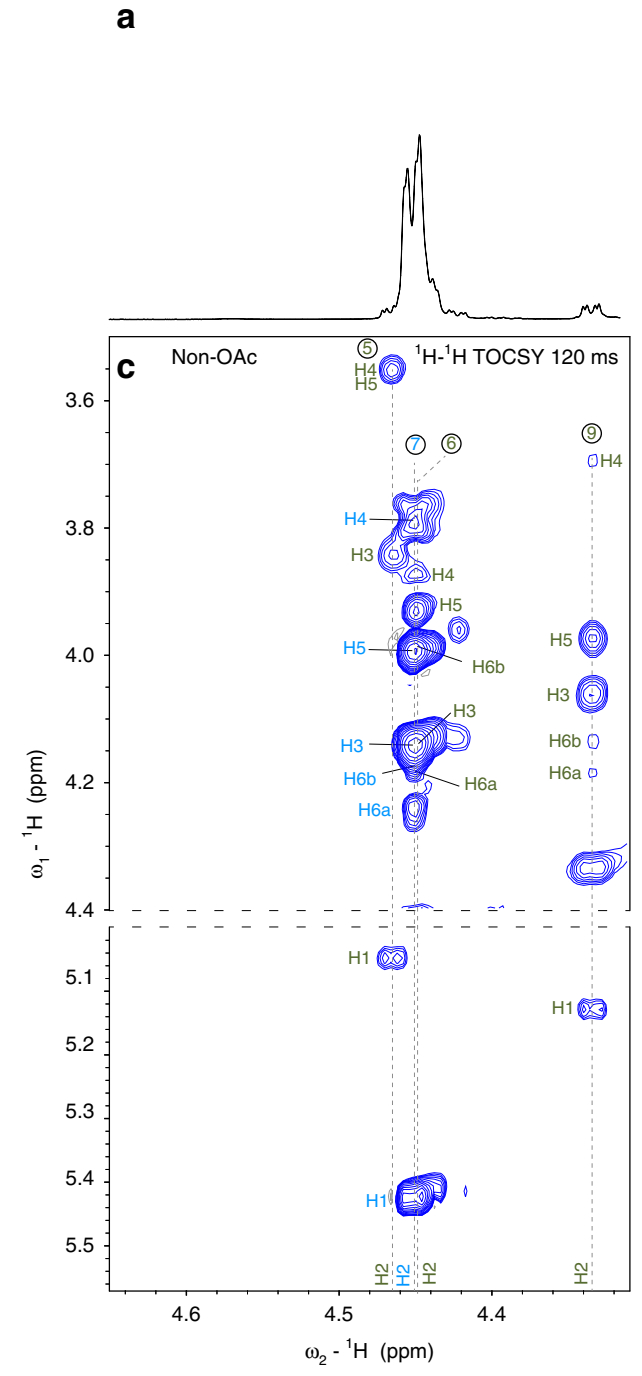

Spin systems present in both samples

(7) ...-P $\square^{\alpha} \square^{\alpha} \mathrm{P}-\ldots \quad \alpha-M a n N A c-6-P$ (internal)

(9) ...-P $\square^{-6} \mathrm{OH} \quad \alpha-$ ManNAc-6-P at reducing end

(5) ...-P $\square^{\beta} \mathrm{OH} \quad \beta-\mathrm{ManNAC}-6-\mathrm{P}$ at reducing end

(6) $\mathrm{P}^{6} \square^{\alpha} \mathrm{P}-\ldots \quad \alpha-$ ManNAC-6-P at non-reducing end

Major internal moieties (1)(3)(7)

Minor internal moieties (2)(4)

Terminal moieties b
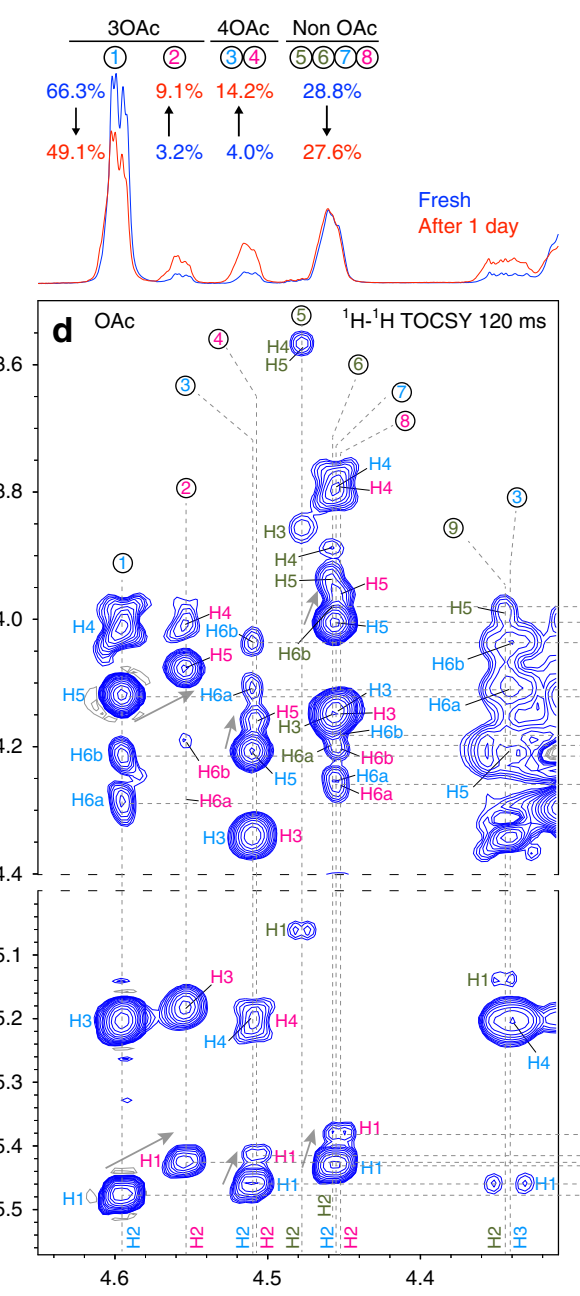
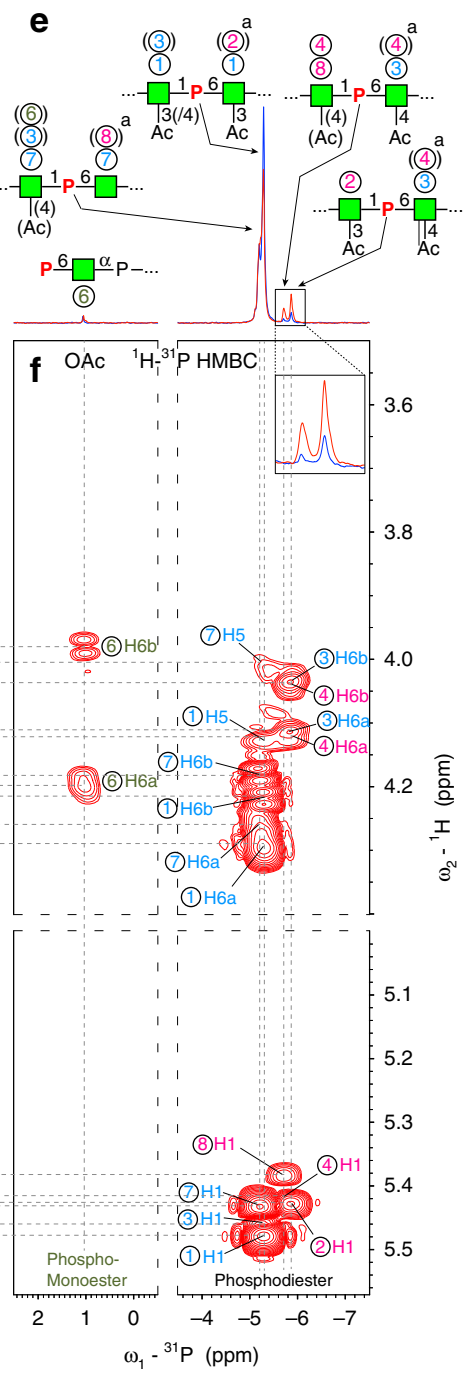
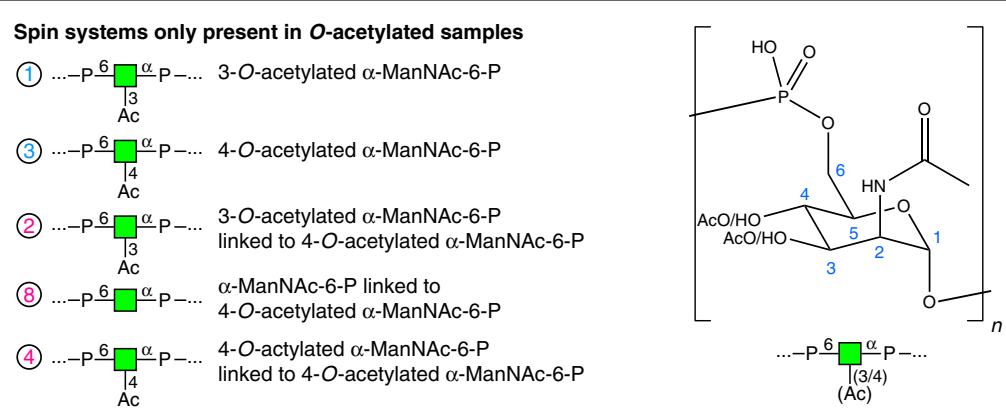

Fig. 2 Comprehensive NMR analysis of $\boldsymbol{O}$-acetylated and non- $\boldsymbol{0}$-acetylated serogroup $\mathbf{A}$ polymer. $\mathbf{a}, \mathbf{b}^{1} \mathrm{H}$ NMR spectra of non- $\mathrm{O}$-acetylated (a) and $\mathrm{O}$-acetylated (b) serogroup $\mathrm{A}$ capsule polymer. b Freshly purified $\mathrm{O}$-acetylated polymer (blue) was incubated at $45^{\circ} \mathrm{C}$ and $\mathrm{pH} 7$ for one day (red), leading to a shift in $\mathrm{O}$-acetylation from $\mathrm{C} 3$ to $\mathrm{C} 4 . \mathbf{c}, \mathbf{d} 2 \mathrm{D}^{1} \mathrm{H}-{ }^{1} \mathrm{H}$ TOCSY (total correlation spectroscopy) spectra showing the $\mathrm{H} 2$ signal of each spin system on the $\omega 2$ axis and the correlations between $\mathrm{H} 2$ and the other ManNAc protons on the $\omega 1$ axis. Four spin systems were identified in the non- $\mathrm{O}$-acetylated sample (c) and nine in the $O$-acetylated sample (d). The identified spin systems were labeled 1 to 9 , with the most downfield spin system labeled 1 and the most upfield labeled 9. e ${ }^{31} \mathrm{P}$ NMR of $\mathrm{O}$-acetylated polymer. aindicates spin systems that belong to moieties, which cannot be excluded as connecting residue. $\mathbf{f}^{1} \mathrm{H}-{ }^{31} \mathrm{P} \mathrm{HMBC}$ spectrum corresponding to the $1 \mathrm{D}^{31} \mathrm{P}$ spectrum shown in $\mathbf{e}$.

considerably slower (Supplementary Fig. 2). Interestingly, the observed $O$-acetyl migration induced changes in the ${ }^{1} \mathrm{H}$ and ${ }^{31} \mathrm{P}$ NMR spectra (Fig. 2e, f; approximately -5.73 and $-5.88 \mathrm{ppm}$ ) that were incongruent with previous assignments ${ }^{14,19}$. To address these discrepancies, we repeated the experiment and performed a comprehensive characterization of the $O$-acetylation pattern by 2D NMR (Fig. 2c-f).
Two-dimensional (2D) NMR analysis of $O$-acetylated NmACPS. A complete de novo assignment was performed starting from the considerably less complex non-O-acetylated polymer (compare Fig. 2a, $c$ with Fig. 2b, d). To increase signals resulting from the polysaccharide termini, we used partially hydrolyzed CPS, an approach that facilitated the assignment of the corresponding spin systems (Supplementary Fig. 3). A well-resolved part of a $2 \mathrm{D}^{1} \mathrm{H}^{-1} \mathrm{H}$ 
TOCSY (total correlation spectroscopy) spectrum was chosen (Fig. 2c, d) that shows the $\mathrm{H} 2$ signal of each spin system on the $\omega_{2}$ axis and the correlations between $\mathrm{H} 2$ and the other ManNAc protons on the $\omega_{1}$ axis. A total of nine spin systems were identified (four in the non-O-acetylated and nine in the $O$-acetylated sample, with the most downfield and upfield systems in Fig. 2d labeled 1 and 9, respectively (Supplementary Tables 1-3). Our data confirm the previous assignment of the major spin systems 1,3 and 7, which belong to 3-O-acetylated, 4-O-acetylated and non-O-acetylated repeating units, respectively ${ }^{7}$.

The well-isolated, less abundant spin system 2 has been, so far, ambiguously assigned to either a 3,4-di-O-acetylated ManNAc ${ }^{14}$ or a 3 OAc-ManNAc being connected to a 4 OAc-ManNAc at its reducing end ${ }^{7}$. Spin system 2 shows correlations similar to those observed for 1 (3OAc-ManNAc), including the characteristic $\mathrm{H} 3$ chemical shift of $5.18 \mathrm{ppm}$, indicating $\mathrm{O}$-acetylation at C3 (Fig. 2d and Supplementary Fig. 4). However, 1 and 2 differ by their H5 and $\mathrm{H} 1$ resonances (Fig. 2d, see arrows) and by the well-resolved ${ }^{31} \mathrm{P}$ chemical shifts of the $\mathrm{C} 1$-linked phosphodiester $(-5.28$ versus $-5.88 \mathrm{ppm}$, Fig. $2 \mathrm{f}$ ). The latter finding suggests that 1 and 2 are quite similar, but connected to different neighboring units at their reducing ends. Following the vertical dotted lines of both ${ }^{31} \mathrm{P}$ chemical shifts $(-5.28 \mathrm{ppm}$ for $1,-5.88 \mathrm{ppm}$ for 2$)$ and searching for correlations to the neighboring unit, reveals crosspeaks to characteristic $\mathrm{H6a} / \mathrm{H} 6 \mathrm{~b}$ signals (Fig. 2f). The ${ }^{31} \mathrm{P}$ resonance of the $\mathrm{C} 1$-linked phosphodiester of 2 correlates with $\mathrm{H} 6 \mathrm{a} / \mathrm{H} 6 \mathrm{~b}$ of 3 (4OAc-ManNAc), whereas the ${ }^{31} \mathrm{P}$ resonance of the phosphodiester of 1 correlates to $\mathrm{H} 6 \mathrm{a} / \mathrm{H} 6 \mathrm{~b}$ of 1 (3OAc-ManNAc). Consequently, 1 belongs to a $3 \mathrm{OAc}-\mathrm{ManNAc}$ that is linked at its reducing end to another 3OAc-ManNAc, whereas 2 refers to 3OAcManNAc linked to 4OAc-ManNAc. Owing to chemical shift degeneracies, a smaller population of 1 following nonOAcManNAc cannot be excluded. Importantly, the chemical shifts observed for $\mathrm{H} 3 / \mathrm{H} 4$ (Fig. 2d) and C3/C4 (Supplementary Figs. 5 and 6) of 1 and 2 are almost identical, clearly excluding 4-O-acetylation of 2 and thus 3,4-di-O-acetylation on the same ManNAc moiety.

We observed two weak spin systems, namely 4 and 8 , that had not been described before. They show similar chemical shifts to the overlapping major spin systems 3 (4OAc-ManNAc) and 7 (nonOAc-ManNAc) (Fig. 2d), but differ by their $\mathrm{H} 5$ and $\mathrm{H} 1$ chemical shifts, reminiscent of the differences between 1 and 2 . The unique $\mathrm{H} 1$ chemical shift of $8(5.381 \mathrm{ppm})$ shows a clear correlation to the unique ${ }^{31} \mathrm{P}$ resonance at $-5.72 \mathrm{ppm}$, which in turn correlates with $\mathrm{H} 6 \mathrm{a} / \mathrm{H} 6 \mathrm{~b}$ of 3 (4OAc-ManNAc, Fig. 2f). Thus, 8 is nonOAc-ManNAc linked to 4 OAc-ManNAc at its reducing end. In accordance, 4 seems to be 4 OAc-ManNAc linked to $4 \mathrm{OAc}-\mathrm{ManNAc}$ at its reducing end. Unfortunately, the $\mathrm{H} 1$ resonance of 4 overlaps partially with other $\mathrm{H} 1$ resonances, preventing the identification of an unambiguous connection to the reducing end neighboring moiety. Also, the $\mathrm{H} 2-\mathrm{H} 6 \mathrm{a} / \mathrm{H} 6 \mathrm{~b}$ correlations of 4 are not clearly visible and likely hidden under the stronger $\mathrm{H} 2-\mathrm{H} 6 \mathrm{a} / \mathrm{H} 6 \mathrm{~b}$ correlations of 3 , making it difficult to deduce the connection to its non-reducing end moiety. However, since 3 and 4 belong to 4 OAc-ManNAc moieties that differ only by the preceding neighbor, it is likely that the resonances of $\mathrm{H6a} /$ $\mathrm{H} 6 \mathrm{~b}$ are very similar. In summary, all signals of internal repeating units are present in a major $(1,3$, and 7$)$ and in a minor form $(2,4$, and 8$)$. The major forms represent units linked to 3OAcManNAc or nonOAc-ManNAc at their reducing ends, while the minor forms are linked to $4 \mathrm{OAc}-\mathrm{ManNAc}$. The quantification of the distinct structural motifs is presented as Supplementary Note 1.

Spin systems 5 and 9 were present in both samples (Fig. 2c, d) and were assigned to the $\beta$ - and $\alpha$-anomer of non- $O$-acetylated ManNAc at the reducing end, respectively, based on the similarity to published chemical shifts of free ManNAc ${ }^{20}$ (Supplementary Table 1). Spin system 6 originates from a terminal ManNAc with a phosphomonoester at C6 and represents the non-reducing end of the polymer as shown by a distinct ${ }^{31} \mathrm{P}$ chemical shift of $0.63 \mathrm{ppm}$ correlating to $\mathrm{H6a} / \mathrm{H} 6 \mathrm{~b}$ of 6 in a ${ }^{1} \mathrm{H}_{-}{ }^{31} \mathrm{P} \mathrm{HMBC}$ (heteronuclear multiple bond correlation) spectrum (Fig. $2 \mathrm{f}$ and Supplementary Fig. 7).

Importantly, all termini identified in the $O$-acetylated sample resulted from nonOAc-ManNAc, indicating that hydrolysis of the $O$-acetylated polymer occurred exclusively between non- $O$ acetylated residues, suggesting their linkage to be less stable.

$\boldsymbol{O}$-Acetylation increases the stability of NmA-CPS. Next, we analyzed the stability of $O$-acetylated and non- $O$-acetylated polymer under conditions that mimicked (i) hydrolysis during vaccine manufacturing $\left(80^{\circ} \mathrm{C}, \mathrm{pH} 4.7\right.$, Fig. 3a) and (ii) storage of vaccines in tropical climate $\left(45^{\circ} \mathrm{C}, \mathrm{pH} 7\right.$, Fig. $\left.3 \mathrm{~b}\right)$. Samples were taken at the indicated time-points and analyzed using Alcian blue/silver-stained polyacrylamide gel electrophoresis (PAGE). After both treatments, the $\mathrm{O}$-acetylated polymer migrated slower than the non-O-acetylated polymer, indicating higher molecular mass and, therefore, higher stability. To confirm this finding, we quantified the change in the average degree of polymerization (DP) according to an established method $^{19}$ that determines the average DP from the ratio between internal phosphodiesters and terminal phosphomonoesters obtained by ${ }^{31} \mathrm{P}$ NMR (Fig. 2e). Again, OAc-CPS exhibited higher stability (Fig. 3c).

Overall structure of CsaC. To provide insight into the structural and mechanistic basis of CPS $O$-acetylation, we solved the structure of wild type CsaC (CsaC-WT, $2.0 \AA$ resolution) in its apo state as well as complexes of CsaC-H228A with acetyl-CoA (2.0 ̊ resolution) and CsaC-WT with a tetrasaccharide fragment of the natural acceptor substrate (CPS-DP4, $1.95 \AA$ resolution) (Supplementary Table 4). Consistent with the oligomeric state of CsaC in solution (Supplementary Fig. 8), the crystal packing of all CsaC structures contains a tetrameric assembly with two protein molecules in the asymmetric unit (Fig. 4a). Each protomer consists of a central eight-stranded $\beta$-sheet that is sandwiched by six $\alpha$-helices and capped by a lid area formed by helices $\alpha 5, \alpha 6$, and a7 (Fig. 4b, c). Contact between the protomers is mainly mediated by helices $\alpha 3$ and $\alpha 7$ (Fig. 4a and Supplementary Fig. 9). A deep positively charged groove, which traverses the protein surface (Fig. 4e, f), allows substrate binding and opens into an active site that is characterized by a catalytic triad composed of S114, H228 and D198 (Fig. 5a). A DALI search ${ }^{21}$ revealed structural homology of $\mathrm{CsaC}$ with serine ester hydrolases of the $\alpha / \beta$-hydrolase $(\mathrm{ABH})$ fold superfamily, a large protein family that encompasses mainly hydrolytic enzymes ${ }^{22-24}$, as exemplified with esterase A from Streptococcus pyogenes (Supplementary Fig. 10, $Z$-score 18.4).

CoA binding in the acetylated H228A(-AcS114)-CoA complex. As soaking of CsaC-WT crystals with acetyl-CoA or CoA yielded only incomplete substrate density and co-crystallization trials were unsuccessful, we soaked crystals of the catalytically impaired variant $\mathrm{H} 228 \mathrm{~A}$ with acetyl-CoA. All protomers showed complete density for CoA with a free SH-group (Fig. 4e and Supplementary Fig. 11a). Concomitantly, the triad serine showed an extended electron density with a contour indicative of its $\mathrm{O}$-acetylated form (AcS114) (Fig. 5b and Supplementary Fig. 11a). This suggests that CsaC adopted the double displacement mechanism of $\mathrm{ABH}$ esterases $^{24}$ and forms a transient acetyl-enzyme intermediate that 
a

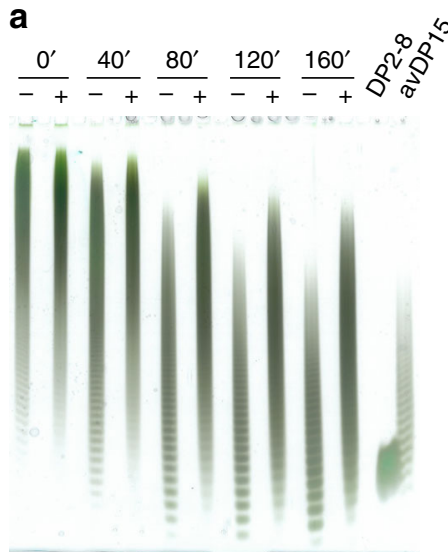

b

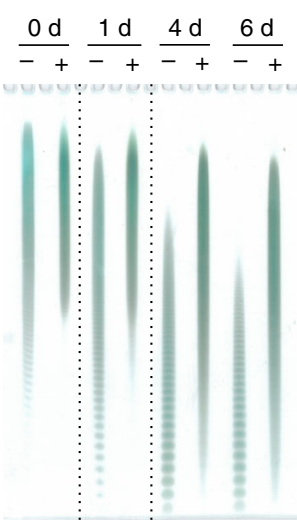

C

\begin{tabular}{c|c|c} 
C & \multicolumn{2}{|c}{$\begin{array}{c}\text { Average degree } \\
\text { of polymerization }\end{array}$} \\
\hline Days & + & - \\
\hline 0 & 96 & 96 \\
1 & 69 & 40 \\
4 & 29 & 14 \\
6 & 20 & 10
\end{tabular}

Fig. 3 Stability of NmA-CPS. a, b Non-O-acetylated ( - ) and enzymatically $O$-acetylated $(+)$ capsule polymer was subjected to mild acidic hydrolysis in acetate buffer $\mathrm{pH} 4.7$ at $80^{\circ} \mathrm{C}$ a and phosphate buffer $\mathrm{pH} 7.0$ at $45^{\circ} \mathrm{C} \mathbf{b}$ to simulate the hydrolysis conditions during vaccine manufacturing and prolonged storage in tropical climate, respectively. Samples were taken at the indicated time-points and the experiment was continued until an average degree of polymerization (avDP) of 15 was reached, which reflects the avDP used for the generation of anti-NmA glycoconjugate vaccines ${ }^{17,68}$. Partially hydrolyzed polymers were separated by PAGE and visualized by Alcian blue/silver staining. An oligomer mix with avDP15 and a mixture of short oligomers containing dimers to octamers (DP2-8) were used as markers ${ }^{17}$. Source data are provided as a Source Data file. c The avDP of $O$-acetylated ( + ) and non$\mathrm{O}$-acetylated (-) polymer was monitored by ${ }^{31} \mathrm{P}$ NMR under conditions corresponding to the experiment shown in $\mathbf{b}$. According to a previously published method $^{19}$, avDP values were calculated from ${ }^{31} \mathrm{P}$ NMR signals and expressed as $\left(P_{\mathrm{Int}} / P_{\mathrm{Ter}}\right)+1$, where $P_{\mathrm{Int}}$ is the molar concentration of the internal phosphate groups (phosphodiester groups) and $P_{\text {Ter }}$ is the molar concentration of terminal phosphate groups (phosphomonoester groups).

a

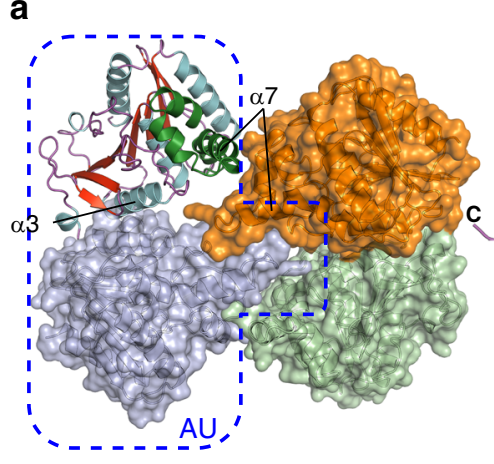

b

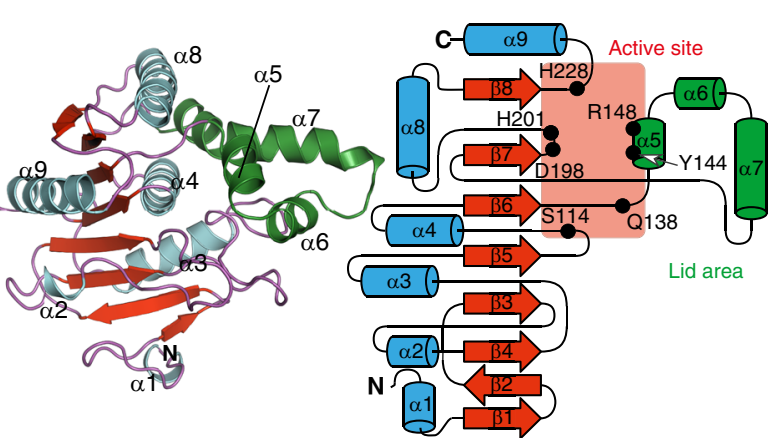

d

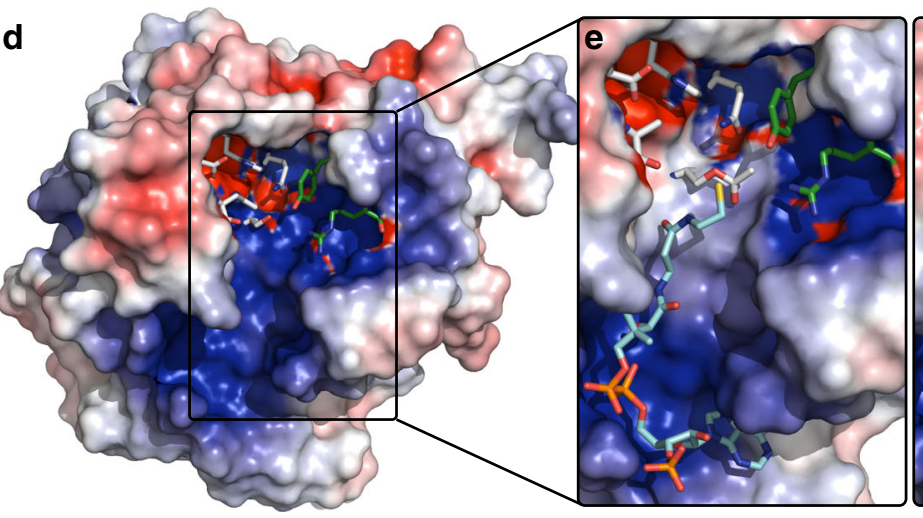

C

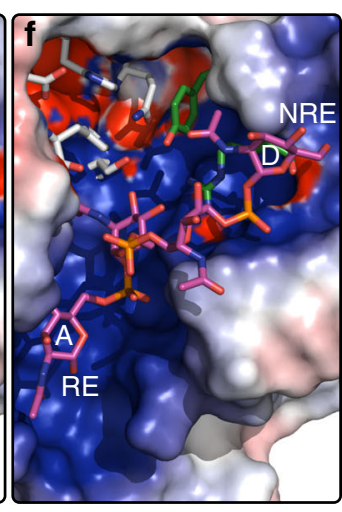

Fig. 4 Crystal structure of CsaC. a Tetrameric assembly of $\mathrm{CsaC}$ with the contents of the asymmetric unit in blue outline. b Secondary structure arrangement of the $\mathrm{CsaC}$ protomer. c Secondary structure topology map of CsaC protomer with important active site residues marked. $\mathbf{d}$-f Electrostatic surface potential (blue-positive, red-negative, white-neutral) with active site residues in stick representation of ligand-free wild type CsaC protomer (d), acetyl-CoA-soaked CsaC-H228A (e), and CPS-DP4-soaked wild type CsaC (f). Pyranose ring A at the reducing end (RE) and pyranose ring D at the non-reducing (NRE) end of the CPS-DP4 are highlighted.

is trapped in the absence of H228 (Supplementary Fig. 12). CoA, as the first reaction product, is bound along the positively charged groove in an L-shaped conformation (Fig. 4e). The pantetheine moiety is engaged in hydrophobic contacts (I233, A39, and F40), and only the distal carbonyl oxygen is specifically recognized by S113-O $\gamma$, a contact that directs the SH-group towards the triad serine S114 (Supplementary Fig. 13). The purine nucleobase of CoA is sandwiched between I11 and K48 and locked into position 


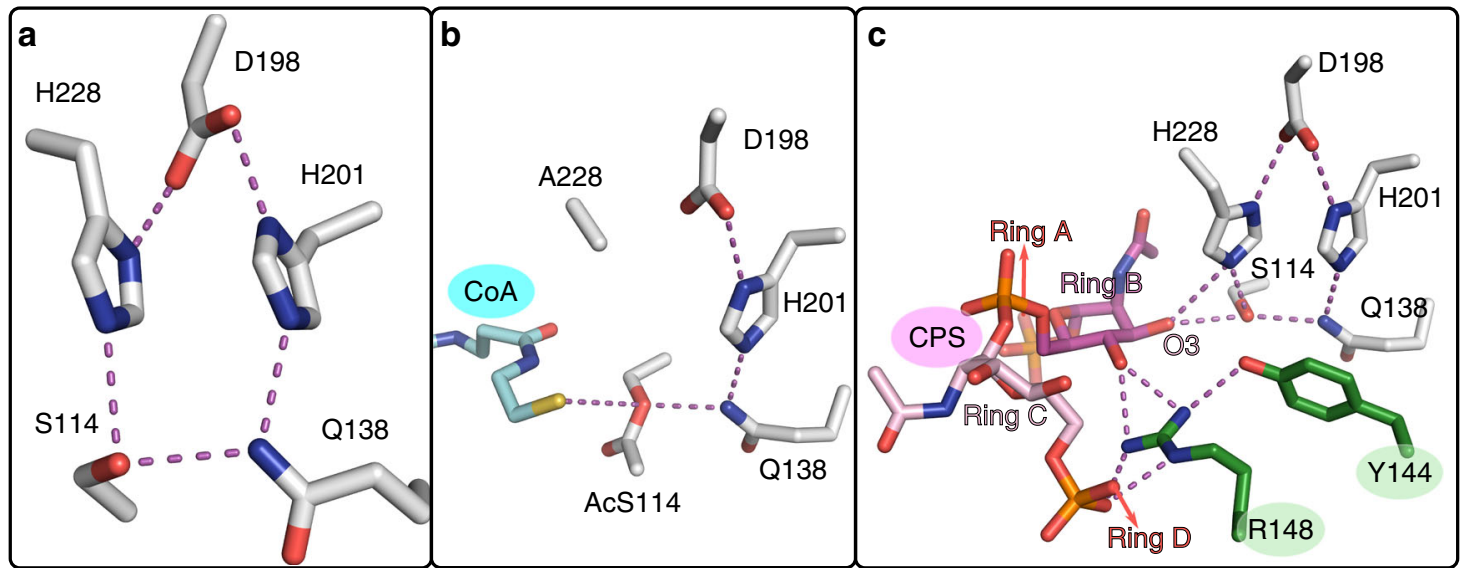

d

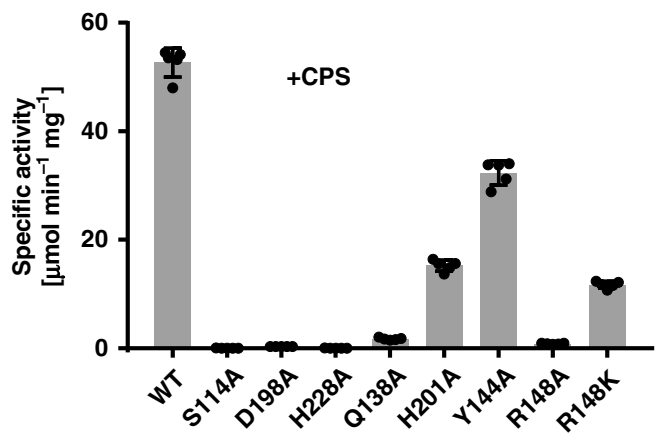

e

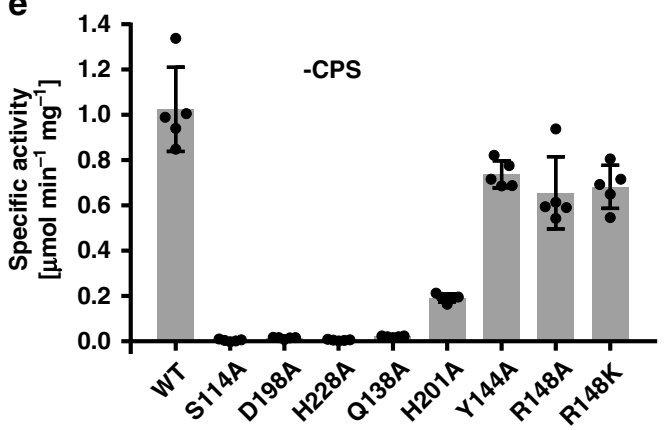

f

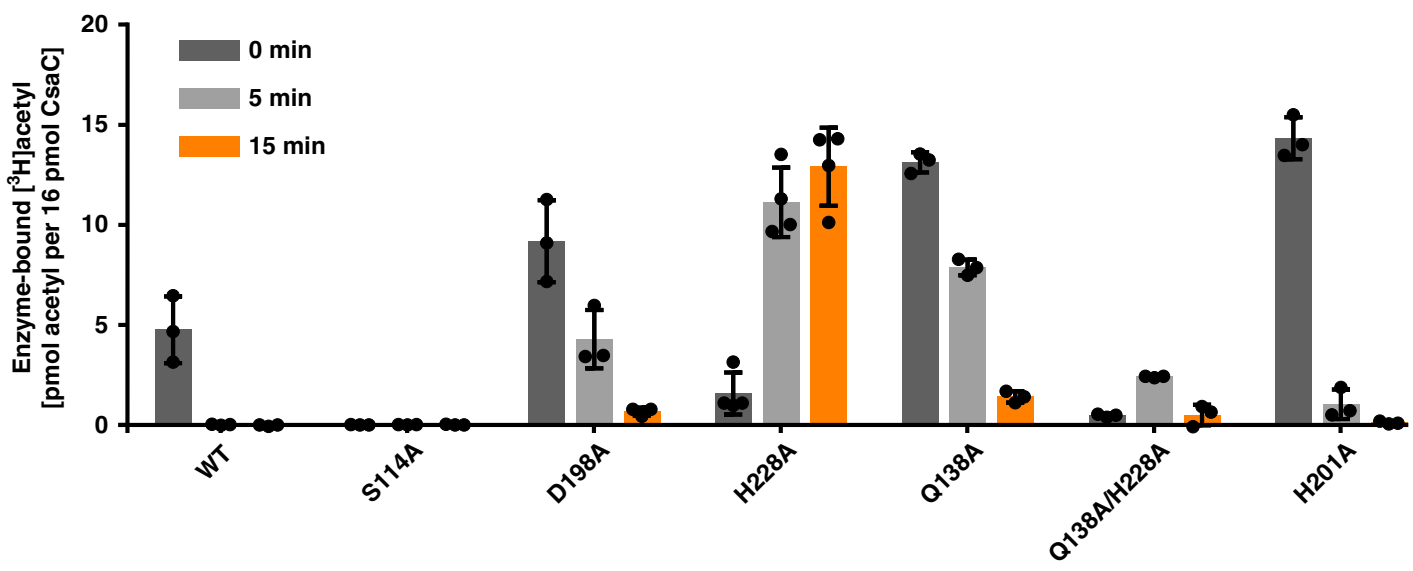

Fig. 5 Active site description of CsaC. a-c Active site residues with hydrogen-bond network (distances $\leq 3.2 \AA$ ) for native wild type $C$ saC a, acetyl-CoAsoaked CsaC-H228A b, and CPS-DP4-soaked wild type CsaC c. d Transferase activity of wild type CsaC and active site mutants in presence of CPS (mean $\pm \mathrm{SD}, n=5$ independent experiments). e Hydrolase activity towards acetyl-CoA measured in the absence of CPS (mean $\pm S D, n=5$ independent experiments). $\mathbf{f}$ Detection of acetyl-enzyme intermediates. Incorporation of radioactively labeled acetyl groups from $\left[{ }^{3} \mathrm{H}\right]$ acetyl-CoA was measured in the absence of CPS (mean \pm SD, $n=4$ independent experiments for CsaC-H228A and $n=3$ independent experiments for CsaC-WT and all other variants). Source data are provided as a Source Data file.

by a hydrogen bond between adenine-N7 and Y49-N, while the phosphate oxygens of the ADP- $3^{\prime}$-phosphate moiety are encased by a hydrogen network provided by R237, K53, Y51 and I52 (Fig. 4e and Supplementary Fig. 13).

The acceptor binding site accommodates four ManNAc moieties. To investigate the structural basis of acceptor binding, crystals of CsaC-WT were soaked with a CPS fragment consisting of four ManNAc moieties (CPS-DP4), which is sufficiently long to serve as acceptor substrate (Supplementary Fig. 14). For the complete tetrasaccharide, the electron density is found in the positively charged groove (Fig. $4 \mathrm{f}$ and Supplementary Figs. 11b and 15). An assessment of the substrate electron densities of DP4, DP5, and DP6 soaked crystal structures revealed no significant difference in dimension and location. Since data statistics and quality of the electron density map were best for the DP4 data set, further analysis was performed on these data. A high-affinity acceptor binding site can be identified that accommodates four ManNAc moieties, designated $\mathrm{A}$ to $\mathrm{D}$ from reducing to nonreducing end. While the tetrasaccharide traverses the active site with the non-reducing end pointing towards the lid domain, the internal ManNAc moiety $\mathrm{B}$ faces the reactive center. The binding site of ManNAc moiety A and B overlaps with that of the pantetheine arm of CoA (Fig. 4e, f and Supplementary Fig. 15), indicating that $\mathrm{CoA}$ is released prior to acceptor binding. 
Contacts between acceptor and $\mathrm{CsaC}$ are mainly facilitated by residues $\mathrm{K} 43, \mathrm{~S} 113, \mathrm{~S} 114, \mathrm{R} 148$, and $\mathrm{H} 228$ (the latter three are illustrated in Fig. 5c and Supplementary Fig. 16).

CsaC shows an unconventional active site architecture. CsaC harbors a serine-histidine-aspartate triad composed of S114, $\mathrm{H} 228$, and D198, which occur in the topological arrangement of the canonical $\mathrm{ABH}$-fold, with $\mathrm{S} 114$ being part of a nucleophilic elbow motif ( $\left.\mathrm{GX}^{114} \mathrm{SXG}\right)$, a typical feature of $\mathrm{ABH}$-enzymes that places the nucleophile at the tip of a sharp turn 22,24 . In CsaC, however, the classical triad is amended by two additional residues, H201 and Q138, yielding a bifurcated network of hydrogenbond donors and acceptors with D198 as bifurcation point and the amino acid pairs H228/S114 and H201/Q138 as the two arms (Fig. 5a). Beyond the catalytic center, the H201/Q138 arm is extended by Y79 (Supplementary Fig. 17), which allows precise positioning of Q138 by engaging its $\mathrm{N} \varepsilon 2$ and $\mathrm{O} \varepsilon 1$ via hydrogenbonding with the flanking $\mathrm{H} 201-\mathrm{N} \varepsilon 2$ and $\mathrm{Y} 79-\mathrm{OH}$, respectively. In this unusual active site arrangement, $\mathrm{S} 114-\mathrm{O} \gamma$ is in hydrogenbond distance not only to $\mathrm{H} 228-\mathrm{N} \varepsilon 2$ as in a classical triad, but also to Ne2 of Q138, which appears to be a unique element of CsaC. In the H228A(-AcS114)-CoA complex, Q138 positions AcS114, which is approached on the opposite side by the SHgroup of $\mathrm{CoA}$ (Fig. 5b). In the CsaC-CPS complex, the acceptor $\mathrm{O} 3$ of ring $\mathrm{B}$ is in $\mathrm{H}$-bond distance to $\mathrm{S} 114-\mathrm{O} \gamma$ and $\mathrm{H} 228-\mathrm{Ne} 2$ (Fig. 5c). The latter is, thus, well-positioned to act as general base deprotonating $\mathrm{O} 3$. In contrast, and similar to the situation in the H228A(-AcS114)-CoA complex, Q138 is located opposite to the substrate entry site and, thus, lacks direct substrate interactions (Fig. 5c). The catalytic unit of $\mathrm{CsaC}$ is accomplished by an oxyanion hole provided by the backbone amides of F40 and K115 (Supplementary Fig. 18), suggesting that the CsaC-catalyzed transfer mechanism involves oxyanion-containing tetrahedral intermediates as proposed in Supplementary Fig. 12.

Enzymatic activity of WT and mutant forms of CsaC. CsaCWT shows high transfer activity towards CPS and a 52-fold lower transfer activity towards water (measured as enzyme-dependent hydrolysis of acetyl-CoA in the absence of CPS) (Fig. 5d, e). As expected, single alanine replacement of the triad residues S114 and $\mathrm{H} 228$ resulted in a complete abrogation of detectable transfer activity, and a D198A exchange reduced transfer activity by $99.5 \%$ $(+\mathrm{CPS})$ and $98.6 \%$ (-CPS). However, a severe drop in activity by 96.8\% (+CPS) and 98.0\% (-CPS) was also seen for Q138A, revealing a substantial contribution of this unconventional active site residue to catalysis. Exchange of $\mathrm{H} 201$, involved in the positioning of Q138, reduced transfer activity by over 70\%. An R148A replacement extinguished transfer activity towards CPS, but not towards water, substantiating a critical role of R148 in CPS placing. The loss of activity towards CPS is only partially rescued by an R148K exchange (Fig. 5d) as only the guanidinium function allows the dual H-bonding with two acceptor sites, as seen in the CsaC-CPS complex (Fig. 5c and Supplementary Fig. 16). Replacement of Y144, which is H-bonded with R148, abolished transfer activity by $38.6 \%$ (+CPS) and $28.0 \%$ (-CPS).

To monitor the formation of the transient acetyl-enzyme intermediate, we incubated CsaC-WT in the presence of $\left[{ }^{3} \mathrm{H}\right]$ acetyl-CoA. Radiolabeled protein was detected directly after mixing and quickly disappeared thereafter due to hydrolysis of the acetyl-enzyme adduct and complete turnover of acetyl-CoA (Fig. 5f). Covalent adduct formation with S114 was confirmed by liquid chromatography-electrospray ionization-tandem mass spectrometry, while with both methods no adduct was seen for CsaC-S114A (Fig. 5f and Supplementary Figs. 19-22). CsaCD198A, -Q138A and $-\mathrm{H} 201 \mathrm{~A}$ rapidly formed a covalent intermediate, but showed decelerated adduct hydrolysis compared to CsaC-WT (Fig. 5f). CsaC-H228A, in contrast, showed no detectable adduct hydrolysis, demonstrating an essential function of $\mathrm{H} 228$ in the second half-reaction, i.e., transfer of the acetyl group from AcS114 to the acceptor (CPS or water). However, how is the acetyl adduct formed by CsaC-H228A in the first half-reaction, in which the triad histidine usually enables the adduct formation by increasing the nucleophilicity of the catalytic serine? In CsaC, the only other residue that could fulfill this function is Q138, assuming the basicity of its amide function was increased, e.g., by amide-imidic acid tautomerism as proposed in Supplementary Fig. 12b. A similar imine-mediated pathway for proton elimination is discussed for $\zeta$-fold prenyltransferases ${ }^{25}$ and the imidic form of an amide side chain as part of a proton transfer network has been directly shown for endoglucanase by neutron crystallography ${ }^{26}$. In support of our hypothesis that Q138 can substitute H228 as a general base in the first halfreaction, Q138 is optimally positioned in both CsaC-WT and the CsaC-H228A(-AcS114)-CoA complex (Supplementary Fig. 23) and impaired acetyl-adduct formation was seen upon simultaneous replacement of $\mathrm{H} 228$ and Q138 (Fig. 5f). In the second half-reaction, Q138 is too distant from the CPS acceptor to substitute H228. Yet, the transfer activity of CsaC-Q138A is severely impaired, suggesting a critical role of Q138 in optimal positioning of AcS114 for the incoming nucleophile and stabilization of a subsequently formed tetrahedral intermediate (Supplementary Figs. 12a and 18b, c).

Structural basis of regioselective $\boldsymbol{O}$-acetylation of CPS. Analysis of the CsaC-CPS complex revealed that the bound tetrasaccharide adopts a step-like conformation with the central phosphodiester bridge dividing the molecule into a reducing disaccharide and a non-reducing disaccharide half (Fig. 6). Specific interactions with $\mathrm{CsaC}$ are limited to pyranose ring $\mathrm{B}$ and the phosphodiester bridge interconnecting ring $\mathrm{C}$ and $\mathrm{D}$. The orientation of ring $\mathrm{B}$ within the substrate entry site allows precise positioning of $\mathrm{O} 3$ for nucleophilic attack (Figs. $5 \mathrm{c}$ and 6 ). Selective access of the $3-\mathrm{OH}$ to the reactive center is ensured by involving the flanking functional groups in acceptor-enzyme interactions. While the carbonyl oxygen of the $\mathrm{C} 2 \mathrm{~N}$-acetyl group is in hydrogen bonding with the hydroxyl group of S113, the C4 hydroxyl group is engaged in a bifurcated H-bond with R148 (Figs. 5c and 6; and Supplementary Fig. 16). Anchoring of the guanidinium group of R148 by two flanking H-bonds (with Y144 and the phosphodiester between ring $\mathrm{C}$ and $\mathrm{D})$ directs the central R148-O4 interaction away from the catalytic center, thus ensuring regioselective attack of $\mathrm{O} 3$.

\section{Discussion}

Native NmA-CPS on intact cells as well as purified polymer contain $\mathrm{O}$-acetyl groups in position $\mathrm{O} 3$ and $\mathrm{O} 4$ of the ManNAc repeating units ${ }^{27}$. As genetic deletion of the $\mathrm{O}$-acetyltransferase $\mathrm{CsaC}$ resulted in a complete loss of CPS $O$-acetylation ${ }^{14}$, it was previously concluded that $\mathrm{CsaC}$ has dual specificity for $\mathrm{O} 3$ and $\mathrm{O} 4$ and, thus, allows 3-O-, 4-O- and even 3,4-di-O-acetylation of the ManNAc units ${ }^{14,27}$. However, our data demonstrate strict regioselectivity of $\mathrm{CsaC}$ for $\mathrm{O} 3$ and uncovered 4-O-acetylation as a secondary effect due to acetyl migration between cis-diols, which may proceed via a cyclic orthoester derivative ${ }^{28}$. The nonenzymatic shift from $\mathrm{O} 3$ to $\mathrm{O} 4$ occurred upon the storage of 3-Oacetylated CPS and yielded an $\mathrm{O}$-acetylation pattern $(56.8 \% \mathrm{O} 3$ and $13.9 \%$ O4) comparable to the natural polymer ${ }^{5,7}$. The phenomenon of $O$-acetyl group migration is well known in the context of sialic acids ${ }^{7,29,30}$. Consistent with our findings, $O$ acetyl group migration observed in the polysialic acid CPS of 


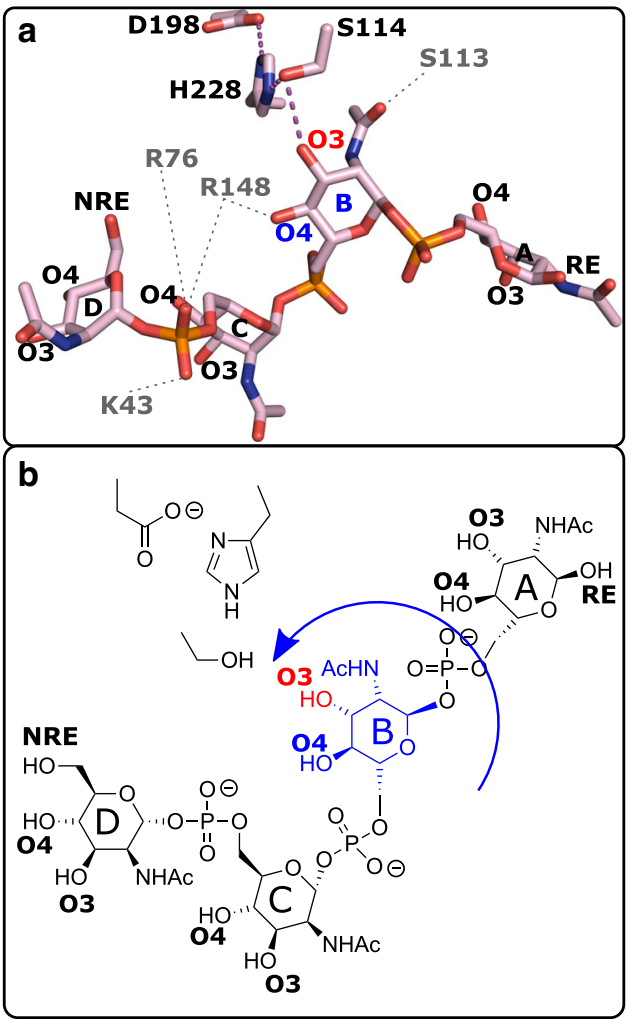

Fig. 6 Orientation of CPS-DP4 rings A-D. Crystal structure of wild-type $\mathrm{CsaC}$ soaked with CPS-DP4 (a) and schematic representation (b). O3 (red) of ring $B$ (blue) is in physical proximity to the catalytic triad serine 114 , while O4 protrudes away from the active site. Hydrogen bond (dashed magenta lines) distances are $\leq 3.2 \AA$. Gray dashed lines indicate coordination by amino acid residues depicted in gray single-letter code. Amino acid residues of the catalytic triad are labeled in black.

$\mathrm{NmC}$ occurred over a period of days when monitored in water ${ }^{7}$ and migration rates of free sialic acids increased at $\mathrm{pH}$ values above 6 , with high rates and partial $O$-acetyl group losses at $\mathrm{pH}$ values above $7.5^{29,30}$. Considering that CPS harvest and purification is performed under neutral to mild basic conditions, the varying levels of 3-O- and 4-O-acetylation that have been reported for $\mathrm{NmA}-\mathrm{CPS}^{5,7,27}$ might mainly reflect variations in the CPS processing protocols.

Our comprehensive 2D NMR analysis highlighted the importance of multiple correlation spectra to assign the complex NMR signals of the NmA-CPS, which were attributed to nine different structural motifs. The NMR data exclude the presence of 3,4-di$O$-acetylated ManNAc units and helped to overcome ambiguities that led to misassignments in the past ${ }^{14}$. The newly acquired knowledge allows precise quantification of the $O$-acetylation level on the basis of eight spin systems (Supplementary Note 1) and contributes to improved quality control of vaccine formulations ${ }^{31}$.

Owing to the chemical lability of the anomeric phosphodiester linkage in aqueous solution, the NmA-CPS is the least stable of the meningococcal polysaccharides ${ }^{4,19}$. Efforts to generate stable glycomimetics yielded, so far, only compounds that are less immunogenic than the natural epitope $\mathrm{e}^{32-34}$. However, approaches to improve their antigenic properties are ongoing ${ }^{35-38}$, and in this context site-selective, enzymatic $O$-acetylation might provide a valuable tool.

Our results show that $O$-acetylation significantly increases the stability of the NmA CPS and that hydrolysis of anomeric phosphodiester linkages occurs exclusively between non- $O$ acetylated ManNAc units. Comparison with the more stable NmX-CPS $([\rightarrow 4)$ GlcNAc- $(1-\mathrm{P} \rightarrow] \mathrm{n})$, which contains GlcNAc instead of ManNAc and lacks $O$-acetylation, suggests that the axial orientation of the $\mathrm{C} 2 \mathrm{~N}$-acetyl group of ManNAc favors carbonyl-assisted intramolecular hydrolysis of the anomeric phosphodiester linkage in the NmA-CPS ${ }^{19}$. The introduction of an electron-withdrawing $\mathrm{O}$-acetyl group in $\mathrm{C} 3$ of ManNAc will mitigate the attack of the carbonyl oxygen in $\mathrm{C} 2$, which might explain the increased stability of $O$-acetylated NmA-CPS. Moreover, molecular dynamics simulations predicted that $O$-acetylation shifts the conformational preference of NmA-CPS from a compact to an extended form ${ }^{36}$. Thus, $O$-acetylation may not only increase CPS stability, but also define the polysaccharide dimensions and, thus, the thickness of the capsule. In summary, and together with our finding that $\mathrm{CsaC} \mathrm{O}$-acetylates also shorter oligosaccharides, our data offer new possibilities for using CsaC as tool for optimized, site-selective $O$-acetylation (i) to increase the stability of size-reduced NmA-CPS used for glycoconjugate vaccines, which are currently produced under harsh conditions that may cause a partial loss of $O$-acetyl groups $(O$-acetylation levels can be as low as $61.5 \%{ }^{39}$ ) and (ii) to increase the immunogenicity of non-native glycomimetics, which show improved stability, but are less immunogenic than the native $\mathrm{NmA}^{-\mathrm{CPS}^{32}}$.

Our structural analysis of $\mathrm{CsaC}$ identified the capsule $O$-acetyltransferase of $\mathrm{NmA}$ as an $\alpha / \beta$-fold enzyme with Ser-His-Asp triad. The Ser-His-Asp triad occurs in several distinct folds, including the $\mathrm{ABH}$-fold, subtilisin fold, chymotrypsin fold and SGNH-fold, and is mainly seen as core catalytic machinery in hydrolytic enzyme reactions ${ }^{40,41}$. So far, several bacterial cell wall $O$-acetyltransferases with a Ser-His-Asp triad have been crystallized and all present an SGNH- or SGNH-like fold ${ }^{42-45}$. The crystal structure of $\mathrm{CsaC}$ revealed that this polysaccharide $\mathrm{O}$ acetyltransferase adopts an $\mathrm{ABH}$-fold and, thus, clearly differs from the above mentioned transferases (see Supplementary Figs. 24 and 25 for comparison). The large ABH-fold superfamily contains primarily hydrolases, but also encompasses members with other catalytic functions ${ }^{22,46}$. Based on a central ABH-fold as scaffold for a catalytic triad, functional variability can arise from additional structural elements. Insertions in the C-terminal part frequently form lid structures that restrict access to the catalytic center and/or shape the substrate-binding pocket ${ }^{46}$. In CsaC, the lid area provides R148, the pivotal residue in acceptor positioning, which critically contributes to acceptor-substrate binding and regioselectivity.

The identification of an acetyl-serine adduct indicates that CsaC follows the canonical mechanism of $\mathrm{ABH}$-esterases, a double-displacement bi-bi mechanism involving two tetrahedral oxyanion intermediates $^{22}$. Different from esterases, however, the CsaC-mediated reaction involves a sugar hydroxyl group instead of water for cleavage of the acetyl-enzyme intermediate during the second half-reaction (Supplementary Fig. 12a). So far, structural information on ABH-transferases is largely limited to enzymes that are listed in the ESTHER database ${ }^{47}$ as members of the homoserine transacetylase (HAT) subfamily. Like CsaC, HATs transfer acetyl groups from acetyl-CoA to a hydroxyl group, but use L-homoserine, L-serine, or deacetylcephalosporin C as acceptor substrates ${ }^{48-50}$. Despite sharing a Ser-His-Asp triad and catalyzing similar reactions, HATs and CsaC show closer structural homology to different subsets of $\mathrm{ABH}$-esterases than to each other and differ in the fine structure of their active sites. HATs not only lack the His201-Q138-Y79-based triad extension seen in CsaC, but also show a different main-chain conformation of the oxyanion loop. In HATs, this conformation is distinct from esterases to lower the activity of water in the active site ${ }^{22}$. CsaC, by contrast, shows an "activating" esterase-like conformation 
(Supplementary Fig. 26) and substantial hydrolysis of the acetylenzyme adduct was observed in the absence of CPS. Thus, in the acceptor-bound state, the optimal geometry of the enzymesubstrate complex might be key to favor the acceptor hydroxyl over water. This affords precise positioning of both acceptor hydroxyl and acetyl-serine adduct, which is mediated by R148 and Q138, respectively, the two non-triad residues that are critical for CsaC-mediated CPS O-acetylation. Notably, Q138 is highly conserved in several uncharacterized bacterial proteins with a nucleophile elbow motif (Supplementary Fig. 27 and Supplementary Data 1), suggesting that the unusual active site arrangement of $\mathrm{CsaC}$ is shared by a panel of putative $\mathrm{ABH}$-fold enzymes of unknown function. Future analyses of these enzymes may show, whether extension of the Ser-His-Asp triad by an extra glutamine evolved as a general strategy to transform $\mathrm{ABH}$-fold esterases into transferases.

\section{Methods}

Cloning and purification. The generation of the plasmid pStrep-CsaC-His was described previously ${ }^{16}$. For the generation of $\mathrm{pCsaC}-\mathrm{His}$, csac was amplified using the primers AB90/FF57 (Supplementary Table 5) and pStrep-CsaC-His as template. The resulting PCR product was cloned back into pStrep-CsaC-His via NdeI/ XhoI, leading to the loss of the Strep tag. All mutations were introduced using the primers shown in Supplementary Table 5 and the QuikChange ${ }^{\oplus}$ XL Site-Directed Mutagenesis Kit (Stratagene) according to the manufacturer's guidelines. Constructs were expressed in Escherichia coli BL21(DE3) and purified on an ÄKTA FPLC (GE Healthcare) via their C-terminal His-tag using immobilized nickel affinity chromatography with $50 \mathrm{mM}$ Tris $\mathrm{pH} 8.0,300 \mathrm{mM} \mathrm{NaCl}, 20 \mathrm{mM}$ imidazole as binding buffer and $50 \mathrm{mM}$ Tris $\mathrm{pH} 8.0,300 \mathrm{mM} \mathrm{NaCl}, 500 \mathrm{mM}$ imidazole as elution buffer. Protein containing fractions were pooled, transferred into storage buffer (50 mM Hepes pH 7.0, $100 \mathrm{mM} \mathrm{NaCl}, 5 \mathrm{mM} \mathrm{MgCl}, 1 \mathrm{mM}$ EDTA) and snap-frozen in liquid nitrogen ${ }^{16}$.

CsaC-mediated in vitro $\mathbf{0}$-acetylation. $\mathrm{CsaC}$-mediated $\mathrm{O}$-acetylation of either 1 or $20 \mathrm{mg}$ of in vitro synthesized non-O-acetylated polymer was performed for $4 \mathrm{~h}$ at $37^{\circ} \mathrm{C}$ in the presence of $0.8 \mu \mathrm{M} \mathrm{CsaC}$ and the indicated concentration of acetylCoA (sodium salt, GERBU) (Supplementary Fig. 1) in a total volume of $0.5 \mathrm{~mL}$ $(1 \mathrm{mg})$ or $10 \mathrm{~mL}(20 \mathrm{mg})$ of acetylation buffer $(25 \mathrm{mM}$ Tris, $\mathrm{pH} 7.5,50 \mathrm{mM} \mathrm{NaCl})$. The modified polymer was purified by anion exchange chromatography (AEC) using a MonoQ HR 5/5 column or a MonoQ 10/100 GL column (both GE Healthcare) and a linear sodium chloride gradient. CPS containing fractions eluting at $500-600 \mathrm{mM} \mathrm{NaCl}$ were pooled, dialyzed against water (ZelluTrans, Roth, $1 \mathrm{kDa}$ molecular mass cutoff) and freeze-dried for NMR analysis ${ }^{16}$

Nuclear magnetic resonance spectroscopy. All spectra were recorded on a $600 \mathrm{MHz}$ Avance III HD spectrometer (Bruker Biospin, Germany) equipped with a ${ }^{1} \mathrm{H} /{ }^{13} \mathrm{C} /{ }^{15} \mathrm{~N} /{ }^{31} \mathrm{P}$-QXI probe at $298 \mathrm{~K}$. Typically, lyophilized samples were dissolved in $500 \mu \mathrm{L} \mathrm{D}_{2} \mathrm{O}(100 \% \mathrm{D}$, Armar, Leipzig, Germany) and transferred to standard $5 \mathrm{~mm}$ tubes (type TA, Armar, Leipzig, Germany). Concentrations ranged from $1 \mathrm{mg} / \mathrm{mL}$ to $5 \mathrm{mg} / \mathrm{mL}$. One-dimensional ${ }^{1} \mathrm{H}$ spectra were recorded with 64 scans, recycle delays of $2 \mathrm{~s}, 65536$ points and a spectral width of $20 \mathrm{ppm}$ resulting in a measurement time of $5 \mathrm{~min}$. One-dimensional ${ }^{31} \mathrm{P}$ spectra were recorded using 352 scans, a recycle delay of $3 \mathrm{~s}, 16384$ points and a spectral width of $50.1 \mathrm{ppm}$ resulting in a measurement time of $22 \mathrm{~min}$. Two-dimensional (2D) ${ }^{1} \mathrm{H}-{ }^{1} \mathrm{H}$ COSY spectra were acquired with the pulse sequence cosygpppf (Bruker library) using 32 scans, a recycle delay of $2 \mathrm{~s}, 2048 \times 128$ points and spectral widths of $8.33 \times$ $8.33 \mathrm{ppm}$ with a duration of $\sim 2 \mathrm{~h}$. Standard $2 \mathrm{D}^{1} \mathrm{H}^{-1} \mathrm{H}$ TOCSY spectra were measured using a mixing time of $80 \mathrm{~ms}, 4$ scans, a recycle delay of $2 \mathrm{~s}, 2048 \times 512$ points and spectral widths of $8.33 \times 8.33 \mathrm{ppm}$ with a duration of $1 \mathrm{~h} 30 \mathrm{~min}$. Twodimensional (2D) ${ }^{1} \mathrm{H}-{ }^{13} \mathrm{C}$ HSQC spectra were recorded using hsqcedetgpsisp2.2 (Bruker library) with 32 scans, a recycle delay of $1.5 \mathrm{~s}, 2048 \times 230$ points and spectral widths of $16.0 \times 100.4 \mathrm{ppm}$ with a duration of $3 \mathrm{~h} 30 \mathrm{~min}$. Twodimensional (2D) ${ }^{1} \mathrm{H}^{13} \mathrm{C}$ HMBC spectra were recorded using the pulse sequence hmbcgpndqf (Bruker library) with 64 scans, a recycle delay of $2 \mathrm{~s}, 4096 \times 512$ points and spectral widths of $20.02 \times 222.4 \mathrm{ppm}$ with a duration of $20 \mathrm{~h} 30 \mathrm{~min}$. Twodimensional (2D) ${ }^{1} \mathrm{H}^{-31} \mathrm{P}$ HMBC spectra were acquired using the pulse sequence hmbclpndqf (Bruker library) with 32 scans, a recycle delay of $1.5 \mathrm{~s}, 4096 \times 64$ points and spectral widths of $10.0 \times 30.5 \mathrm{ppm}$ with a duration of $1 \mathrm{~h} 8$ min unless stated otherwise. Spectra were processed with Topspin 3.6.1 (Bruker Biospin, Germany) and analyzed with Sparky 3.115 (Goddard T.D., Kneller D.G. (2008) SPARKY 3. University of California, San Francisco, CA). The ${ }^{1} \mathrm{H}$ resonances were referenced to DSS (4,4-dimethyl-4-silapentane-1-sulfonic acid) using an external sample of $2 \mathrm{mM}$ sucrose containing $0.5 \mathrm{mM}$ DSS (standard sample for water suppression from Bruker Biospin, Germany). ${ }^{13} \mathrm{C},{ }^{15} \mathrm{~N}$, and ${ }^{31} \mathrm{P}$ axis were referenced indirectly according to IUPAC-IUBMB-IUPAB ${ }^{51}$ using the chemical shift referencing ratios of $0.251449530,0.101329118$, and 0.404808636

Generation of CPS-derived oligosaccharides with defined DP. Serogroup A oligosaccharides of defined DP were produced by partial hydrolysis of the phosphodiester bond, starting from enzymatically synthesized, non-O-acetylated serogroup A polymer ${ }^{17}$. The polymer was incubated in sodium acetate buffer $(50 \mathrm{mM}$ sodium acetate, $\mathrm{pH} 4.8$ ) for $6 \mathrm{~h}$ at $73^{\circ} \mathrm{C}$, yielding oligosaccharides that carry a phosphomonoester at the non-reducing end ${ }^{19,52}$, which could be removed by calf intestinal alkaline phosphatase ${ }^{16,53}$. Obtained oligosaccharides were purified by AEC on an ÄKTA FPLC system (GE Healthcare) equipped with a MonoQ HR 5/5 column (GE Healthcare). $\mathrm{H}_{2} \mathrm{O}$ and $1 \mathrm{M} \mathrm{NaCl}$ were used as mobile phases $\mathrm{M} 1$ and $\mathrm{M} 2$, respectively, at a flow rate of $1 \mathrm{~mL} / \mathrm{min}$. Samples were separated by consecutive linear gradient steps $(0-5 \% \mathrm{M} 2$ over $1 \mathrm{~mL}, 5-20 \% \mathrm{M} 2$ over $10 \mathrm{~mL}$, and $20-30 \% \mathrm{M} 2$ over $20 \mathrm{~mL}$ ). Acetate and GlcNAc-1P were used to calibrate the column. The amount of oligosaccharide in each fraction was estimated from the peak area obtained at $214 \mathrm{~nm}$ under the assumption that each ManNAc residue contributes equally to the absorbance of the respective oligomer. Fractions were dialyzed against water (ZelluTrans, Roth, 1-kDa molecular mass cutoff) and freeze-dried, and equal concentrations were adjusted with water. Identity and DP of the sizefractionated oligosaccharides were confirmed by HPLC-AEC and HPAEC-PAD (high-performance anion exchange chromatography with pulsed amperometric detection). HPLC-AEC was performed on a Prominence UFLC-XR system (Shimadzu) equipped with a CarboPac PA-100 column (Dionex, Thermo Fisher Scientific). Samples were separated at $50^{\circ} \mathrm{C}$ with $\mathrm{H}_{2} \mathrm{O}$ and $1 \mathrm{M} \mathrm{NaCl}$ as mobile phases M1 and M2, using a -2 curved gradient from 0 to $30 \%$ M2 over 4 min followed by a linear gradient from 30 to $84 \%$ M2 over $33 \mathrm{~min}^{54}$ (Supplementary Fig. 14a). LCsolution version $1.25 \mathrm{SP} 4$ (Shimadzu) was used for data collection and analysis. HPAEC-PAD ${ }^{19}$ was performed on an ICS 5000 system (Dionex, Thermo Fisher Scientific) equipped with a CarboPac PA200 column (Thermo Fisher Scientific) calibrated with ManNAc and ManNAc-6P (Carbosynth) (Supplementary Fig. 14b). Samples were separated at $30^{\circ} \mathrm{C}$ with $\mathrm{H}_{2} \mathrm{O}, 1 \mathrm{M} \mathrm{NaNO}_{3}$ and $500 \mathrm{mM} \mathrm{NaOH}$ as mobile phases M1, M2 and M3, using a constant concentration of $20 \%$ of M3 and a linear gradient from 1 to $16 \%$ of M2 over 50 min. Chromeleon 7.2 SR4 (Thermo Scientific) was used for data collection and analysis.

CPS stability assay. To analyze the stability of $O$-acetylated and non- $O$-acetylated NmA-CPS, $3.9 \mathrm{mg}$ of CPS were dissolved in $105 \mu \mathrm{L}$ of $50 \mathrm{mM}$ sodium acetat buffer $\mathrm{pH}$ 4.7 and incubated at $80^{\circ} \mathrm{C}$. After the indicated time points (Fig. $3 \mathrm{a}$ ), $15 \mu \mathrm{L}$ of sample were neutralized at room temperature with $0.4-0.6 \mu \mathrm{L}$ of $1 \mathrm{M} \mathrm{NaOH}$ and supplemented with $15 \mu \mathrm{L}$ of $2 \mathrm{M}$ sucrose. Oligomers were separated by high percentage (25\%) PAGE performed at $4{ }^{\circ} \mathrm{C}$ and $400 \mathrm{~V}$ and stained with $0.5 \%$ Alcian blue and $0.6 \%$ silver nitrate ${ }^{16,17,55}$. Samples shown in Fig. 3b were prepared as described below for Fig. 3c and analyzed as described above for Fig. 3a. For ${ }^{31} \mathrm{P}$ NMR analysis (Figs. 3c), $4.8 \mathrm{mg}$ of NmA-CPS were dissolved in $525 \mu \mathrm{L}$ of $57 \mathrm{mM}$ potassium phosphate buffer $\mathrm{pH} 7$ and incubated at $45^{\circ} \mathrm{C}$. The $\mathrm{pH}$ was monitored before and after the experiment ( $\mathrm{pH} 6.96$ for non-OAc- and $\mathrm{pH} 6.80$ for OAc-CPS). The avDP values were calculated from ${ }^{31} \mathrm{P}$ NMR signals of internal phosphate groups (phosphodiester groups) and terminal phosphate groups (phosphomonoester groups) ${ }^{19}$.

Analysis of acetyl group migration. O-Acetylated NmA-CPS (1.6 mg) was dissolved in $550 \mu \mathrm{L} \mathrm{D} \mathrm{D}_{2} \mathrm{O}$ (final pH of 5.6) or $550 \mu \mathrm{L}$ potassium phosphate buffer (final $\mathrm{pH}$ of 7.0) and incubated for the indicated time period (Fig. $2 \mathrm{~b}$ and Supplementary Fig. 2).

0-Acetyltransferase assay. Enzymatic activity of purified $\mathrm{CsaC}$ variants was determined in a spectrophotometric assay with 5,5-dithio-bis-(2-nitrobenzoic acid) (DTNB). The reaction was performed at $30^{\circ} \mathrm{C}$ in a total volume of $100 \mu \mathrm{L}$ containing $50 \mathrm{mM}$ Tris-HCl pH 7.5, $50 \mathrm{mM} \mathrm{NaCl}, 20 \%$ glycerol, 2 mM DTNB (Sigma) and $1 \mathrm{mM}$ acetyl-CoA (sodium salt, GERBU) with or without $160 \mu \mathrm{g} / \mathrm{mL}$ non-Oacetylated NmA-CPS. The reaction was initiated by adding $16 \mathrm{pmol}$ of purified enzyme. A reaction without enzyme was performed as blank to account for spontaneous hydrolysis of acetyl-CoA. The generation of $\mathrm{CoA}$ was monitored by reaction of its free sulfhydryl group with DTNB, which yields a mixed disulfide and 5-thionitrobenzoic acid. The latter was measured continuously at $405 \mathrm{~nm}$ in halfarea 96-well plates (Greiner) using a PowerWave 340 microtiter plate spectrophotometer (BioTek). The change in optical density at $405 \mathrm{~nm}$ was related to a standard curve obtained for free CoA (Sigma).

Radioactive incorporation assay. To monitor the formation of acetyl-enzyme adducts, $2.5 \mu \mathrm{g}$ of purified $\mathrm{CsaC}$ variants were incubated at $37^{\circ} \mathrm{C}$ with $5 \mu \mathrm{M}$ $(740 \mathrm{~Bq} / \mathrm{pmol})\left[{ }^{3} \mathrm{H}\right]$ acetyl-CoA (American Radiolabeled Chemicals) in $25 \mu \mathrm{L}$ reaction buffer $(50 \mathrm{mM}$ Tris- $\mathrm{HCl} \mathrm{pH} \mathrm{7.5,50} \mathrm{mM} \mathrm{NaCl)}$. The reaction was stopped after 0,5 and $15 \mathrm{~min}$ by spotting $5 \mu \mathrm{L}$ of the reaction mixture (containing 16 pmol $\mathrm{CsaC}$ ) on Whatman $3 \mathrm{MM}$ paper. Free radioactivity was removed by descending paper chromatography in $70 \%$ ethanol containing $300 \mathrm{mM}$ ammonium acetate buffer $\mathrm{pH}$ 7.5. Radioactivity that was incorporated into the chromatographically immobile protein fraction was quantified by scintillation counting. 


\section{Analysis of acetyl-enzyme adducts by liquid chromatography-electrospray} ionization-mass spectrometry (LC-ESI-MS). For detection of acetyl enzyme adducts by mass spectrometry, $2 \mu \mathrm{g}$ of the enzyme ( $64 \mathrm{pmol})$ were mixed with $5 \mu \mathrm{M}$ acetyl-CoA in a final volume of $10 \mu \mathrm{L}$ of reaction buffer $(50 \mathrm{mM}$ Tris- $\mathrm{HCl} \mathrm{pH}$ $7.5,50 \mathrm{mM} \mathrm{NaCl}$ ). The reaction was immediately stopped by adding Laemmli sample buffer, and the reaction mixture was separated by sodium dodecyl sulfate-polyacrylamide gel electrophoresis. Coomassie Blue-stained protein bands were excised and digested with trypsin and Asp-N. Briefly, the gel pieces were dehydrated with acetonitrile and rehydrated with $100 \mathrm{mM} \mathrm{NH} \mathrm{HCO}_{3}$ containing $10 \mathrm{mM}$ dithiothreitol (Roth). Subsequently, gel pieces were treated with $100 \mathrm{mM}$ $\mathrm{NH}_{4} \mathrm{HCO}_{3}$ containing $100 \mathrm{mM}$ iodoacetamide (Sigma). After a second dehydration step with acetonitrile and rehydration with $100 \mathrm{mM} \mathrm{NH}_{4} \mathrm{HCO}_{3}$ buffer followed by dehydration with acetonitrile, the dried gel pieces were rehydrated with $20 \mathrm{ng} / \mu \mathrm{L}$ trypsin (Promega) in $50 \mathrm{mM} \mathrm{NH}_{4} \mathrm{HCO}_{3}$ buffer and incubated overnight at $37^{\circ} \mathrm{C}$. Peptides were extracted with $75 \%$ acetonitrile containing $0.1 \%$ formic acid and dried in a vacuum centrifuge. Peptides were dissolved in $4 \mathrm{ng} / \mu \mathrm{L}$ Asp-N (Promega) in $10 \mathrm{mM}$ Tris- $\mathrm{HCl}$ buffer ( $\mathrm{pH} \mathrm{8.0)}$ ), incubated overnight at $37^{\circ} \mathrm{C}$ and dried again. MS analysis of peptides was performed on a nanoACQUITY UPLC system (Waters) equipped with an analytical column (Waters, BEH130C18, $100 \mu \mathrm{m} \times$ $100 \mathrm{~mm}, 1.7 \mu \mathrm{m}$ particle size) coupled online to an ESI Q TOF (Q TOF Ultima, Waters). Peptides were dissolved in $2 \%$ acetonitrile, $0.1 \%$ formic acid and separated by reverse phase chromatography using acetonitrile as eluent. MS spectra were recorded in positive reflection mode and analytes were automatically subjected to fragmentation (MS/MS). Spectra were analyzed using MassLynx V4.1 software (Waters). MS/MS protein spectra were automatically analyzed using the program ProteinLynx Global Server (Version 2.1, Waters).

CsaC crystallization and structure determination. Native wild type CsaC and CsaC-H228A were crystallized in sitting drop setups at concentrations of $\sim 18 \mathrm{mg} / \mathrm{mL}$. Fine screens around initial screening conditions resulted in many isomorphous crystals. Mother liquor contained $50 \mathrm{mM}$ HEPES pH 7.0 (Applichem), $100 \mathrm{mM}$ HEPES pH 7.6, $100 \mathrm{mM} \mathrm{NaCl}$ (Sigma), $5 \mathrm{mM} \mathrm{MgCl}_{2}$ (Merck), $1 \mathrm{mM}$ EDTA (Merck), and 31-42\% PEG200 (Sigma). Good quality crystals grew at 4,12 , and $18{ }^{\circ} \mathrm{C}$. Structure determination involved de novo phasing by gadoliniumsingle-wavelength anomalous diffraction (SAD) and the obtained CsaC structure was then used as a search model to solve all subsequent structures. Native crystals of CsaC-WT were soaked overnight in mother liquor supplemented with $150 \mathrm{mM}$ Gd-HPDO3A ("caged gadolinium"; Jena Bioscience) at $18^{\circ} \mathrm{C}$. CPS-derived oligosaccharide with DP4 (see Supplementary Fig. 14) was soaked into native wild type crystals overnight at $18^{\circ} \mathrm{C}$. Owing to a lack of an appropriate standard, the absolute concentration of the oligomer remained undetermined. The best relative concentration was determined empirically by soaking different serial dilutions of CPSDP4. Acetyl-CoA was soaked at a concentration of $4 \mathrm{mM}$ into native CsaC-H228A crystals over-night at $18^{\circ} \mathrm{C}$.

Diffraction data (Supplementary Table 4) were measured at the ESRF beamline ID30B and the DESY beamlines P13 and P14. Data were integrated using XDS 56 The Phase problem was solved in crank $2^{57}$. Native and soaked crystal data were solved by Molecular Replacement in Phaser ${ }^{58}$ with the gadolinium-soaked structure as a search model. Structures were refined by several cycles of automatic and manual refinement in Phenix ${ }^{58}$ and $\operatorname{Coot}^{58}$, respectively.

Analytical ultracentrifugation (AUC). AUC experiments were carried out in a Beckman Coulter ProteomeLab XL-I analytical ultracentrifuge with an An-50 Ti rotor at $20^{\circ} \mathrm{C}$ using the absorption scanning optics at $280 \mathrm{~nm}$ and the manufacturer's data acquisition software ProteomeLab XL-I Version 6.0 (Firmware 5.7). The stock solution of CsaC was dialyzed against $50 \mathrm{mM}$ HEPES pH 7.0,0.1 M $\mathrm{NaCl}, 5 \mathrm{mM} \mathrm{MgCl}$, and $1 \mathrm{mM}$ EDTA and diluted with the same buffer. Protein extinction coefficients at $280 \mathrm{~nm}$, partial specific volumes, and buffer viscosities and densities were calculated from amino acid or buffer composition, respectively, by the program SEDNTERP 59 . Protein concentrations were determined spectrophotometrically and are given in monomers (Supplementary Fig. 8). Sedimentation velocity analysis was performed at $35,000 \mathrm{rpm}$ in a concentration range from 3.0 to $40.9 \mu \mathrm{M} \mathrm{CsaC}$ using 3 or $12 \mathrm{~mm}$ double sector centerpieces filled with $100 \mu \mathrm{L}$ or $400 \mu \mathrm{L}$ sample, respectively. For data analysis, a model for diffusion-deconvoluted differential sedimentation coefficient distributions [continuous $\mathrm{c}(\mathrm{s})$ distributions] implemented in the program SEDFIT ${ }^{60}$ was used. Figures were prepared using the program GUSSI ${ }^{61}$

Sedimentation equilibrium experiments were performed at 7000; 9000; 12,000; and $15,000 \mathrm{rpm}$ using $150 \mu \mathrm{L}$ of $9.1 \mu \mathrm{M} \mathrm{CsaC}$ or $40 \mu \mathrm{L}$ of $40.9 \mu \mathrm{M}$ CsaC in standard 12 or $3 \mathrm{~mm}$ double sector centerpieces, respectively. Samples were spun at each rotor speed until no further change in concentration gradient could be observed for at least $8 \mathrm{~h}$. The baseline offset was set to the buffer absorbance measured in each cell near the meniscus after sedimenting the protein for $8 \mathrm{~h}$ at $44,000 \mathrm{rpm}$ at the end of the experiment. The molar mass was determined by globally fitting the data obtained at all rotor speeds and concentrations to a single species model, similar as described previously ${ }^{62}$.

Bioinformatics analyses. Blastp ${ }^{63}$ (protein-protein BLAST) searches were performed against the non-redundant protein sequences database both including and excluding Gram-positive bacteria, yielding hits with $25-40 \%$ sequence identity. Multiple-sequence alignments were performed with Clustal omega ${ }^{64}$ and annotated with Jalview ${ }^{65}$ (Supplementary Fig. 27), and with CLUSTALW implemented in MEGAX $^{66}$ (Supplementary Data 1).

Reporting summary. Further information on research design is available in the Nature Research Reporting Summary linked to this article.

\section{Data availability}

The crystallographic datasets reported herein have been deposited in the PDB repository under accession codes 6YUO, 6YUV, 6YUS, 6YUQ. Source data for Figs. 3, 5 and Supplementary Fig. 14 are provided with this paper. Raw NMR and mass spectrometry data are available from the corresponding authors upon request. Source data are provided with this paper.

Received: 24 July 2020; Accepted: 20 August 2020; Published online: 18 September 2020

\section{References}

1. Peterson, M. E. et al. Meningococcal serogroups and surveillance: A systematic review and survey. J. Glob. Health 9, 010409 (2019).

2. Maiden, M. C. J. The impact of protein-conjugate polysaccharide vaccines: an endgame for meningitis? Philos. Trans. R. Soc. Lond. B. Biol. Sci. 368, 20120147 (2013).

3. Tiffay, K., Jodar, L., Kieny, M.-P., Socquet, M. \& LaForce, F. M. The evolution of the meningitis vaccine project. Clin. Infect. Dis. 61, S396-S403 (2015).

4. Beresford, N. J. et al. Quality, immunogenicity and stability of meningococcal serogroup ACWY-CRM197, DT and TT glycoconjugate vaccines. Vaccine 35 3598-3606 (2017).

5. Bardotti, A. et al. Physicochemical characterisation of glycoconjugate vaccines for prevention of meningococcal diseases. Vaccine 26, 2284-2296 (2008)

6. Bundle, D. R., Smith, I. C. \& Jennings, H. J. Determination of the structure and conformation of bacterial polysaccharides by carbon 13 nuclear magnetic resonance. Studies on the group-specific antigens of Neisseria meningitidis serogroups A and X. J. Biol. Chem. 249, 2275-2281 (1974).

7. Lemercinier, X. \& Jones, C. Full $1 \mathrm{H}$ NMR assignment and detailed O-acetylation patterns of capsular polysaccharides from Neisseria meningitidis used in vaccine production. Carbohydr. Res. 296, 83-96 (1996).

8. Sharma, N., Hanif, S., Rana, R., Upadhyay, D. \& Chhikara, M. K. Evaluation of impact of temperature and $\mathrm{pH}$ alterations on the size and antigenicity of meningococcal serogroup $\mathrm{A}$ and $\mathrm{X}$ polysaccharides and conjugates. Vaccine 37, 965-972 (2019).

9. Liu, T. Y., Gotschlich, E. C., Jonssen, E. K. \& Wysocki, J. R. Studies on the meningococcal polysaccharides. I. Composition and chemical properties of the group A polysaccharide. J. Biol. Chem. 246, 2849-2858 (1971).

10. Jones, C. \& Lemercinier, X. Use and validation of NMR assays for the identity and $\mathrm{O}$-acetyl content of capsular polysaccharides from Neisseria meningitidis used in vaccine manufacture. J. Pharm. Biomed. Anal. 30, 1233-1247 (2002).

11. Lupisan, S. et al. Meningococcal polysaccharide A O-acetylation levels do not impact the immunogenicity of the quadrivalent meningococcal tetanus toxoid conjugate vaccine: results from a randomized, controlled phase III study of healthy adults aged 18 to 25 years. Clin. Vaccin. Immunol. 20, 1499-1507 (2013).

12. Berti, F., De Ricco, R. \& Rappuoli, R. Role of O-acetylation in the immunogenicity of bacterial polysaccharide vaccines. Molecules 23, 1-10 (2018).

13. Berry, D. S., Lynn, F., Lee, C., Frasch, C. E. \& Bash, M. C. Effect of $\mathrm{O}$-acetylation of Neisseria meningitidis serogroup A capsular polysaccharide on development of functional immune responses. Infect. Immun. 70, 3707-3713 (2002).

14. Gudlavalleti, S. K. et al. The Neisseria meningitidis serogroup A capsular polysaccharide O-3 and O-4 acetyltransferase. J. Biol. Chem. 279, 42765-42773 (2004)

15. Harrison, O. B. et al. Description and nomenclature of Neisseria meningitidis capsule locus. Emerg. Infect. Dis. 19, 566-573 (2013).

16. Fiebig, T. et al. Molecular cloning and functional characterization of components of the capsule biosynthesis complex of Neisseria meningitidis serogroup A: toward in vitro vaccine production. J. Biol. Chem. 289, 19395-19407 (2014)

17. Fiebig, T. et al. Efficient solid-phase synthesis of meningococcal capsular oligosaccharides enables simple and fast chemoenzymatic vaccine production. J. Biol. Chem. 293, 953-962 (2018).

18. Lee, H. J. et al. Structural and kinetic characterizations of the polysialic acid O-acetyltransferase OatWY from Neisseria meningitidis. J. Biol. Chem. 284, 24501-24511 (2009). 
19. Berti, F. et al. Relative stability of meningococcal serogroup A and X polysaccharides. Vaccine 30, 6409-6415 (2012).

20. Coxon, B. Deuterium isotope effects in carbohydrates revisited. Cryoprobe studies of the anomerization and NH to ND deuterium isotope induced 13C NMR chemical shifts of acetamidodeoxy and aminodeoxy sugars. Carbohydr. Res. 340, 1714-1721 (2005).

21. Holm, L. \& Rosenström, P. Dali server: conservation mapping in 3D. Nucleic Acids Res. 38, W545-W549 (2010).

22. Rauwerdink, A. \& Kazlauskas, R. J. How the same core catalytic machinery catalyzes 17 different reactions: the serine-histidine-aspartate catalytic triad of $\alpha / \beta$-hydrolase fold enzymes. ACS Catal. 5, 6153-6176 (2015).

23. Ollis, D. L. et al. The alpha/beta hydrolase fold. Protein Eng. 5, 197-211 (1992).

24. Holmquist, M. Alpha/Beta-hydrolase fold enzymes: structures, functions and mechanisms. Curr. Protein Pept. Sci. 1, 209-235 (2000).

25. Malwal, S. R. et al. Catalytic role of conserved asparagine, glutamine, serine, and tyrosine residues in isoprenoid biosynthesis enzymes. ACS Catal. 8, 4299-4312 (2018).

26. Nakamura, A. et al. 'Newton's cradle' proton relay with amide-imidic acid tautomerization in inverting cellulase visualized by neutron crystallography. Sci. Adv. 1, e1500263 (2015).

27. Gudlavalleti, S. K., Szymanski, C. M., Jarrell, H. C. \& Stephens, D. S. In vivo determination of Neisseria meningitidis serogroup A capsular polysaccharide by whole cell high-resolution magic angle spinning NMR spectroscopy. Carbohydr. Res. 341, 557-562 (2006).

28. Dong, H. et al. Stereoelectronic control in regioselective carbohydrate protection. J. Org. Chem. 77, 1457-1467 (2012).

29. Kamerling, J. P. et al. Migration of O-acetyl groups in N,O-acetylneuraminic acids. Eur. J. Biochem. 162, 601-607 (1987).

30. Varki, A. \& Diaz, S. The release and purification of sialic acids from glycoconjugates: methods to minimize the loss and migration of O-acetyl groups. Anal. Biochem. 137, 236-247 (1984).

31. Martini, S. et al. Correction to 'NMR assays for estimating the $\mathrm{O}$-acetyl content of meningococcal polysaccharide serogroup A in quadrivalent conjugate vaccine formulation'. ACS Omega 4, 15771 (2019).

32. Gao, Q. et al. Immunoactivity of protein conjugates of carba analogues from Neisseria meningitidis a capsular polysaccharide. ACS Chem. Biol. 8, 2561-2567 (2013).

33. Fallarini, S. et al. A synthetic disaccharide analogue from Neisseria meningitidis A capsular polysaccharide stimulates immune cell responses and induces immunoglobulin $\mathrm{G}$ (IgG) production in mice when proteinconjugated. ACS Infect. Dis. 1, 487-496 (2015).

34. Torres-Sanchez, M. I. et al. Synthesis and biological evaluation of phosphono analogues of capsular polysaccharide fragments from Neisseria meningitidis A. Chem.-A Eur. J. 13, 6623-6635 (2007).

35. Hlozek, J., Ravenscroft, N. \& Kuttel, M. M. Modeling the conformations of Neisseria meningitidis serogroup a CPS and a carba-analogue: Implications for vaccine development. Carbohydr. Res. 486, 107838 (2019).

36. Hlozek, J., Kuttel, M. M. \& Ravenscroft, N. Conformations of Neisseria meningitidis serogroup $\mathrm{A}$ and $\mathrm{X}$ polysaccharides: the effects of chain length and O-acetylation. Carbohydr. Res. 465, 44-51 (2018).

37. Adamo, R. Advancing homogeneous antimicrobial glycoconjugate vaccines. Acc. Chem. Res. 50, 1270-1279 (2017).

38. Calloni, I. et al. The conformation of the mannopyranosyl phosphate repeating unit of the capsular polysaccharide of Neisseria meningitidis serogroup A and Its carba-mimetic. Eur. J. Org. Chem. 2018, 4548-4555 (2018).

39. Beri, S., Gandhi, D. \& Ravenscroft, N. Use of NMR as an analytical tool in the process development of conjugate vaccines against Haemophilus influenzae type B (Hib) and meningococcal serogroup A (MenA). Biologicals 62, 102-106 (2019).

40. Dodson, G. \& Wlodawer, A. Catalytic triads and their relatives. Trends Biochem. Sci. 23, 347-352 (1998).

41. Mølgaard, A., Kauppinen, S. \& Larsen, S. Rhamnogalacturonan acetylesterase elucidates the structure and function of a new family of hydrolases. Structure 8, 373-383 (2000).

42. Sychantha, D. et al. In vitro characterization of the antivirulence target of Gram-positive pathogens, peptidoglycan O-acetyltransferase A (OatA). PLoS Pathog. 13, 1-26 (2017).

43. Jones, C. S., Sychantha, D., Howell, P. L. \& Clarke, A. J. Structural basis for the $\mathrm{O}$-acetyltransferase function of the extracytoplasmic domain of OatA from Staphylococcus aureus. J. Biol. Chem. 295, 8204-8213 (2020).

44. Riley, L. M. et al. Structural and functional characterization of Pseudomonas aeruginosa $\mathrm{AlgX}$ : role of $\mathrm{AlgX}$ in alginate acetylation. J. Biol. Chem. 288, 22299-22314 (2013).

45. Sychantha, D. et al. PatB1 is an O-acetyltransferase that decorates secondary cell wall polysaccharides. Nat. Chem. Biol. 14, 79-85 (2018).
46. Mindrebo, J. T., Nartey, C. M., Seto, Y., Burkart, M. D. \& Noel, J. P. Unveiling the functional diversity of the alpha/beta hydrolase superfamily in the plant kingdom. Curr. Opin. Struct. Biol. 41, 233-246 (2016).

47. Lenfant, N. et al. ESTHER, the database of the $\alpha / \beta$-hydrolase fold superfamily of proteins: tools to explore diversity of functions. Nucleic Acids Res. 41 D423-D429 (2013).

48. Mirza, I. A., Nazi, I., Korczynska, M., Wright, G. D. \& Berghuis, A. M. Crystal structure of homoserine transacetylase from Haemophilus influenzae reveals a new family of alpha/beta-hydrolases. Biochemistry 44, 15768-15773 (2005).

49. Lejon, S., Ellis, J. \& Valegård, K. The last step in cephalosporin C formation revealed: crystal structures of deacetylcephalosporin $\mathrm{C}$ acetyltransferase from Acremonium chrysogenum in complexes with reaction intermediates. J. Mol. Biol. 377, 935-944 (2008).

50. Oda, K., Matoba, Y., Kumagai, T., Noda, M. \& Sugiyama, M. Crystallographic study to determine the substrate specificity of an L-serine-acetylating enzyme found in the D-cycloserine biosynthetic pathway. J. Bacteriol. 195, 1741-1749 (2013).

51. Markley, J. L. et al. Recommendations for the presentation of NMR structures of proteins and nucleic acids. IUPAC-IUBMB-IUPAB Inter-Union Task Group on the Standardization of Data Bases of Protein and Nucleic Acid Structures Determined by NMR Spectroscopy. J. Biomol. NMR 12, 1-23 (1998).

52. Ricci, S., Bardotti, A., D’Ascenzi, S. \& Ravenscroft, N. Development of a new method for the quantitative analysis of the extracellular polysaccharide of Neisseria meningitidis serogroup A by use of high-performance anionexchange chromatography with pulsed-amperometric detection. Vaccine 19, 1989-1997 (2001).

53. Budde, I. et al. An enzyme-based protocol for cell-free synthesis of natureidentical capsular oligosaccharides from Actinobacillus pleuropneumoniae serotype 1. J. Biol. Chem. 295, 5771-5784 (2020).

54. Litschko, C. et al. A new family of capsule polymerases generates teichoic acidlike capsule polymers in gram-negative pathogens. MBio 9, 16017 (2018).

55. Min, H. \& Cowman, M. K. Combined alcian blue and silver staining of glycosaminoglycans in polyacrylamide gels: Application to electrophoretic analysis of molecular weight distribution. Anal. Biochem. 155, 275-285 (1986).

56. Kabsch, W. XDS. Acta Crystallogr. D. Biol. Crystallogr. 66, 125-132 (2010).

57. Skubák, P. \& Pannu, N. S. Automatic protein structure solution from weak X-ray data. Nat. Commun. 4, 2777 (2013).

58. McCoy, A. J. et al. Phaser crystallographic software. J. Appl. Crystallogr. 40, 658-674 (2007).

59. Laue, T. M., Shah, B. D., Ridgeway, T. M., \& Pelletier, S. L. In Analytical Ultracentrifugation in Biochemistry and Polymer Science. 90-125 (Royal Society of Chemistry, Cambridge, UK, 1992).

60. Schuck, P. Size-distribution analysis of macromolecules by sedimentation velocity ultracentrifugation and lamm equation modeling. Biophys. J. 78, 1606-1619 (2000).

61. Brautigam, C. A. In Methods in Enzymology 562, 109-133 (Academic Press Inc., 2015).

62. Vámosi, G. et al. EGFP oligomers as natural fluorescence and hydrodynamic standards. Sci. Rep. 6, 33022 (2016).

63. Boratyn, G. M. et al. BLAST: a more efficient report with usability improvements. Nucleic Acids Res. 41, W29-W33 (2013).

64. Sievers, F. et al. Fast, scalable generation of high-quality protein multiple sequence alignments using Clustal Omega. Mol. Syst. Biol. 7, 539 (2011).

65. Waterhouse, A. M., Procter, J. B., Martin, D. M. A., Clamp, M. \& Barton, G. J. Jalview Version 2-a multiple sequence alignment editor and analysis workbench. Bioinformatics 25, 1189-1191 (2009).

66. Kumar, S., Stecher, G., Li, M., Knyaz, C. \& Tamura, K. MEGA X: molecular evolutionary genetics analysis across computing platforms. Mol. Biol. Evol. 35, 1547-1549 (2018)

67. Willis, L. M. \& Whitfield, C. Structure, biosynthesis, and function of bacterial capsular polysaccharides synthesized by $\mathrm{ABC}$ transporter-dependent pathways. Carbohydr. Res. 378, 35-44 (2013).

68. Costantino, P. et al. Size fractionation of bacterial capsular polysaccharides for their use in conjugate vaccines. Vaccine 17, 1251-1263 (1999).

\section{Acknowledgements}

We thank Maria Rosaria Romano, Roberto Adamo, Francesco Berti (all GSK vaccines, Siena, Italy), Rita Gerardy-Schahn, Anja Münster-Kühnel, and Françoise Routier (all $\mathrm{MHH}$ ) for helpful discussions and Lidia Litz and Astrid Oberbeck for excellent technical assistance. We also thank the synchrotron staff scientists at the ESRF beamline ID30B and the DESY beamlines P13 and P14 for their help during data collection. This study was funded by Deutsche Forschungsgemeinschaft (DFG, German Research Foundation)project number 262794208; 412824531. 


\section{Author contributions}

T.F. performed and analyzed NMR experiments; and preparative and analytical chromatography. T.F., J.T.C., and P.B. crystallized proteins and collected X-ray data. J.T.C analyzed crystallographic data; solved the crystal structure; and generated structural figures and tables. A.B. performed site-directed mutagenesis and expression experiments; purification of polysaccharides and recombinant proteins; and enzymatic assays. U.C. performed analytical ultracentrifugation analyses. J.I.F. contributed to biochemical data analysis. F.F.R.B. performed and analyzed all MS experiments. U.V. contributed to the design of the project. M.S. performed and analyzed NMR experiments and generated NMR figures. R.F. oversaw the work of P.B.; and contributed to the structure refinement and analysis of crystallographic data. M.M. and T.F. conceived the project; oversaw the work of A.B. and analyzed biochemical data. M.M. analyzed crystallographic data and designed the site-directed mutagenesis approach. M.M., T.F., J.T.C., and M.S. prepared the initial draft of the manuscript and all authors made contributions to the final version.

\section{Funding}

Open Access funding provided by Projekt DEAL.

\section{Competing interests}

The authors declare no competing interests.

\section{Additional information}

Supplementary information is available for this paper at https://doi.org/10.1038/s41467020-18464-y.
Correspondence and requests for materials should be addressed to T.F. or M.M.

Peer review information Nature Communications thanks the anonymous reviewer(s) for their contribution to the peer review of this work.

Reprints and permission information is available at http://www.nature.com/reprints

Publisher's note Springer Nature remains neutral with regard to jurisdictional claims in published maps and institutional affiliations.

(c) (i) Open Access This article is licensed under a Creative Commons Attribution 4.0 International License, which permits use, sharing, adaptation, distribution and reproduction in any medium or format, as long as you give appropriate credit to the original author(s) and the source, provide a link to the Creative Commons license, and indicate if changes were made. The images or other third party material in this article are included in the article's Creative Commons license, unless indicated otherwise in a credit line to the material. If material is not included in the article's Creative Commons license and your intended use is not permitted by statutory regulation or exceeds the permitted use, you will need to obtain permission directly from the copyright holder. To view a copy of this license, visit http://creativecommons.org/ licenses/by/4.0/.

(C) The Author(s) 2020 